\title{
THE JOINT FAR-INFRARED-OPTICAL LUMINOSITY FUNCTION FOR SPIRAL GALAXIES AND DATA FOR THE ABELL 400 AND CANCER CLUSTERS
}

\author{
EDVIGE CORBelli ${ }^{1}$ and EdWin E. SALPETER \\ Center for Radiophysics and Space Research, Space Sciences Building, Cornell University, Ithaca, NY 14853 \\ AND \\ JOHN M. DICKEY \\ Sterrewacht Leiden; and Department of Astronomy, University of Minnesota, Minneapolis, MN 55455 \\ Received 1990 March 15; accepted 1990 September 6
}

\begin{abstract}
The joint far-infrared-optical luminosity function for late-type galaxies, $\Psi$, is examined using an optically selected sample of 183 galaxies from the Revised Shapley-Ames Catalog and from the Virgo Cluster compilation of Helou and coworkers, including peculiar spirals but excluding Sa's. For each of these galaxies we have the far-infrared (FIR) flux at $60 \mu \mathrm{m}, f_{60}$, and at $100 \mu \mathrm{m}, f_{100}$. We show that the distribution of the ratio of FIR to blue luminosity, $r \equiv L_{\text {FIR }} / L_{B}$, depends weakly on $L_{B}$, so that $\Psi$ can be approximated by a function of a single variable, $\psi\left(r^{\prime}\right)$, where $r^{\prime} \equiv r\left(L_{B} / L_{*}\right)^{-\delta}$ with $\delta \simeq 0.08(\simeq 0$ for large $r)$ and $L_{*}$ a constant. The function $\psi\left(r^{\prime}\right)$ is well fitted by a lognormal curve which peaks at $r^{\prime}=0.35$ and has a dispersion of 0.28 . While an excess of galaxies, with respect to the lognormal curve, is visible for large $r^{\prime}$, our optically selected sample of nearby galaxies shows that a population of galaxies with very small values of $r^{\prime}$ is absent. This implies that spiral galaxies with a very low abundance of interstellar dust are rare. We also suggest an explanation for the positive correlation of both $r$ and the FIR color $c\left(c \equiv f_{100} / f_{60}\right)$ with $L_{B}$. By studying the FIR visibility function, we argue that the FIR luminosity function is proportional to $\psi$ for large $r$ and that they both decline with a power law of index $\approx-2$.

Using the Minnesota Automated Plate Scanner, we have an optical list of galaxies as faint as 17th magni tude in the blue in two more distant clusters: the Cancer Cluster and Abell 400. For groups of galaxies, optically similar but individually undetected in the FIR, we build up co-added "class average scans" which can give meaningful class average fluxes as small as $50 \mathrm{mJy}$ at $60 \mu \mathrm{m}$. Using these, we are able to check the distribution function of $r^{\prime}$ at the faint FIR end, even in these more distant clusters.
\end{abstract}

Subject headings: galaxies: clustering - galaxies: photometry — infrared: sources - luminosity function

\section{INTRODUCTION}

Statistical comparison of the optical emission with the farinfrared (FIR) emission of galaxies observed by the Infrared Astronomical Satellite (IRAS) (Neugebauer et al. 1984) depends on the completeness of the catalog from which objects are selected. For nearby galaxy samples the IRAS Point Source Catalog, Version 2 (1988, hereafter PSC), has sufficient sensitivity to determine the FIR luminosity function, $\Phi_{\text {FIR }}$, or the distribution function $\Psi$ of the distance-independent ratio $r=$ $L_{\text {FIR }} / L_{B}$, where $L_{\text {FIR }}$ is the far-infrared luminosity and $L_{B}$ is the blue luminosity, defined below. However, the PSC, being a flux-limited catalog, contains mostly galaxies near the peak of the logarithmic visibility function $V_{\mathrm{FIR}} \propto L_{\mathrm{FIR}}^{5 / 2} \Phi_{\mathrm{FIR}}$. Because of the presence of starburst galaxies, which have high values of $L_{\text {FIR }}$, and of the high power of $L_{\text {FIR }}$ in $V_{\text {FIR }} / \Phi_{\text {FIR }}$, FIR-selected samples tend to be biased toward large values of $r$ (Soifer et al. 1987; Lawrence et al. 1986; Rieke \& Lebofsky 1986). Previous studies (Rowan-Robinson, Helou, \& Walker 1987; Bothun, Lonsdale, \& Rice 1989) have in fact shown the presence of a long tail in the distribution function $\Psi(r)$ toward high $r$-values. We are interested here in the $r$-distribution function, especially for the smallest values of $r$, the opposite end to starburst galaxies. Although this topic has not received much attention, it should give information on whether an appreciable fraction of

\footnotetext{
${ }^{1}$ Postal address: Osservatorio Astrofisico di Arcetri, Largo E. Fermi, 5, 50125 Firenze, Italy.
}

spiral galaxies have extremely low abundances of interstellar dust. We shall discuss the joint luminosity function in $L_{\text {FIR }}$ and $L_{B}$ and, especially, the dependence of the $r$-distribution on $L_{B}$ in terms of the distribution function $\Psi\left(L_{B}, r\right)$, which depends weakly on $L_{B}$.

In order to study small values of $r$ and $L_{B}$ we use optically selected samples of nearby galaxies from the Revised ShapleyAmes Catalog and Virgo Cluster and go deeper in FIR luminosity by using IRAS co-added data to obtain the highest sensitivity. Since cluster galaxies may differ in their FIR properties from field galaxies because of environmental influences (Bicay \& Giovanelli 1987), we also add to the range of cluster environments by studying two additional clusters, an Abell cluster, A400, and the Cancer Cluster. A400 is a relatively spiral-rich cluster and slightly denser than the Virgo Cluster (with X-rays suggesting gas stripping); being rich, it probably has only slight pollution from foreground galaxies. The Cancer Cluster (Bothun et al. 1983) is not a cluster at all but an unbound collection of groups at a similar redshift to A400, and this sample should be intermediate between field galaxies and the Virgo or A400 cluster samples regarding environmental effects. We used the Minnesota Automated Plate Scanner (MAPS) with the Palomar Sky Survey to construct an optical catalog with known completeness properties, and we then obtained IRAS co-added data for these samples. We explain below how we go a step further for these more distant clusters, by obtaining a co-added class average of the FIR luminosity for the less luminous galaxies which remain individually unde- 
tected. We can then check the shape of the luminosity function that we derived using data from more nearby samples.

The organization of the paper is as follows. In $\S 2$ we describe the four separate samples of galaxies we use for the analysis in this paper and the optical and infrared data we have for them. In $\S 3$ we derive the FIR-optical luminosity function, and we discuss it in $\S 4$, together with a model for the FIR luminosity function. We present in $\S 5$ results from the analysis of the A400 and Cancer cluster data, and especially some important information which one can get only from the class average technique. The major results are then summarized in $\S 6$.

\section{THE SAMPLES}

\subsection{The RSA and Virgo Samples}

In order to have optically complete samples of galaxies down to very low optical luminosities, we close two different sets of galaxies from the Revised Shapley-Ames Catalog (Sandage \& Tammann 1981, hereafter RSA), Rb (RSA-bright) and Rf (RSA-faint), and one set of galaxies from the Virgo Cluster (Vo). The samples are defined by the following limits.

$\mathbf{R b}$ and $\mathbf{R f}$ are galaxies from the RSA Catalog which have apparent blue magnitude $B_{T}^{0} \geq 10$ (in order to avoid very extended sources). For the "bright" set, Rb, we allow all Hubble types later than Sab and earlier than $\mathrm{Sd}$, with positions in the range $7^{\mathrm{h}} 30^{\mathrm{m}}<$ R.A. $<11^{\mathrm{h}} 25^{\mathrm{m}}$ and decl. $>0^{\circ}$. For the "fainter" set, Rf, we chose all the later Hubble types Sd, Sm, and Im with decl. $>0^{\circ}$ and without any restriction on R.A. For both sets we make no distinction between barred and unbarred spirals or designations $\mathrm{r}$ or $\mathrm{s}$. We have 87 galaxies in sample $\mathrm{Rb}$, where we included the galaxy NGC 2764 classified as "Amorphous or Sb pec" and NGC 3274 classified as an "S" type galaxy. We have 25 galaxies in sample $\mathrm{Rf}$, leaving out the galaxy NGC 4299, which is in the Virgo Cluster and is included in sample Vo. More than half of these galaxies have reliable detections at 60 and $100 \mu \mathrm{m}$ in the PSC. Taking coordinates from Dressel \& Condon (1976), we obtained onedimensional co-added data from IRAS, processed with the Addscan routine, for all the galaxies which have no source within $3^{\prime}$ in the PSC or correspond to a source with $f_{60}<1.5$ Jy and/or $f_{100}<3.5 \mathrm{Jy}$, where $f_{60}$ and $f_{100}$ are fluxes at 60 and $100 \mu \mathrm{m}$, respectively. The Addscan technique adds all strips of data taken by the IRAS satellite, flipping appropriately so that the resulting scan represents roughly a one-dimensional profile of the sky brightness at the source position. A second-order baseline is removed, and if a tentative signal can be identified, it is fitted by a template function which characterizes the IRAS beam shape.

No IRAS data were available (irrespective of flux) for two source positions in the $R f$ sample and seven in the $\mathrm{Rb}$ sample, so that the $R b$ and $R f$ samples consisted of 80 and 23 galaxies, respectively. The Addscan data, which were made available for the 42 of the 103 galaxies without reliable PSC fluxes, are listed in Table 1 together with the optical data. Note that all 42 sources were detected in both FIR bands. For all galaxies in $\mathrm{Rb}$ and $\mathrm{Rf}$ we used the corrected blue apparent magnitude $B_{T}^{0}$ from the RC2 (de Vaucouleurs, de Vaucouleurs, \& Corwin 1976) when available, instead of the RSA value. We used the distance estimate given in the RSA, rescaled to a Hubble constant of $75 \mathrm{~km} \mathrm{~s}^{-1} \mathrm{Mpc}^{-1}$, to compute the absolute blue magnitude $M_{B}^{0}$. Columns (5) and (6) give the values of $f_{60}$ and $f_{100}$, the flux densities expressed in janskys. All of the 42 sources
TABLE 1

Co-ADDED DATA FOR Rb AND Rf SAMPLE

\begin{tabular}{|c|c|c|c|c|c|c|}
\hline & $\begin{array}{c}\text { Sample } \\
\text { (1) }\end{array}$ & $\begin{array}{c}\text { Name } \\
(2)\end{array}$ & $\begin{array}{l}B_{T}^{0} \\
(3)\end{array}$ & $\begin{array}{c}M_{B}^{0} \\
(4)\end{array}$ & $\begin{array}{r}f_{60} \\
(5)\end{array}$ & $\begin{array}{c}f_{100} \\
(6)\end{array}$ \\
\hline $\mathbf{R b}$ & & NGC 2441 & 12.53 & -21.9 & 1.15 & 4.79 \\
\hline $\mathbf{R b}$ & & NGC 2523 & 12.10 & -22.2 & 0.95 & 3.65 \\
\hline $\mathbf{R b}$ & $\ldots$ & NGC 2541 & 11.73 & -18.8 & 2.28 & 5.19 \\
\hline $\mathbf{R b}$ & & NGC 2545 & 12.67 & -21.4 & 1.00 & 3.25 \\
\hline $\mathbf{R b}$ & $\ldots \ldots$ & NGC 2552 & 12.36 & -18.0 & 0.85 & 2.48 \\
\hline $\mathbf{R b}$ & $\ldots$ & NGC 2713 & 11.99 & -22.4 & 0.96 & 3.53 \\
\hline $\mathbf{R b}$ & $\ldots$ & NGC 2715 & 11.24 & -21.2 & 3.63 & 12.79 \\
\hline $\mathbf{R b}$ & $\ldots$ & NGC 2793 & 12.54 & -20.1 & 0.95 & 2.40 \\
\hline $\mathbf{R b}$ & & NGC 2942 & 12.39 & -22.3 & 0.80 & 2.39 \\
\hline $\mathbf{R b}$ & $\ldots \ldots \ldots \ldots$ & NGC 3184 & 10.18 & -20.3 & 9.75 & 29.36 \\
\hline $\mathbf{R b}$ & & NGC 3254 & 11.64 & -20.3 & 0.77 & 2.94 \\
\hline $\mathbf{R b}$ & $\ldots$ & NGC 3259 & 12.12 & -20.9 & 1.06 & 3.58 \\
\hline $\mathbf{R b}$ & $\ldots \ldots$ & NGC 3274 & 13.25 & -16.7 & 1.16 & 2.30 \\
\hline $\mathbf{R b}$ & ...... & NGC 3287 & 12.32 & -19.6 & 1.86 & 5.73 \\
\hline $\mathbf{R b}$ & & NGC 3319 & 11.38 & -19.6 & 2.08 & 5.74 \\
\hline $\mathbf{R b}$ & $\ldots$ & NGC 3344 & 10.28 & -20.2 & 9.88 & 31.10 \\
\hline $\mathrm{Rb}$ & ............ & NGC 3346 & 11.86 & -19.9 & 1.76 & 6.08 \\
\hline $\mathbf{R b}$ & & NGC 3389 & 11.95 & -19.8 & 4.33 & 10.46 \\
\hline $\mathbf{R b}$ & n........... & NGC 3395 & 12.04 & -20.5 & 5.26 & 9.02 \\
\hline $\mathbf{R b}$ & n......... & NGC 3396 & 12.04 & -20.5 & 5.26 & 9.02 \\
\hline $\mathbf{R b}$ & n.......... & NGC 3403 & 12.07 & -20.2 & 1.44 & 7.24 \\
\hline $\mathbf{R b}$ & $\ldots \ldots \ldots \ldots$ & NGC 3455 & 12.37 & -19.1 & 1.92 & 4.78 \\
\hline $\mathbf{R b}$ & ............. & NGC 3478 & 12.38 & -23.3 & 0.71 & 1.90 \\
\hline $\mathbf{R b}$ & $\ldots \ldots \ldots \ldots$ & NGC 3495 & 11.66 & -19.8 & 3.07 & 9.23 \\
\hline $\mathbf{R b}$ & $\ldots \ldots$ & NGC 3510 & 12.56 & -18.0 & 0.62 & 2.17 \\
\hline $\mathbf{R b}$ & n.......... & NGC 3512 & 12.77 & -19.3 & 1.73 & 4.78 \\
\hline $\mathbf{R b}$ & $\ldots \ldots \ldots \ldots \ldots$ & NGC 3547 & 12.67 & -19.6 & 2.22 & 4.91 \\
\hline $\mathbf{R b}$ & ............. & NGC 3549 & 12.04 & -21.8 & 1.66 & 5.49 \\
\hline $\mathbf{R b}$ & $\cdots$ & NGC 3614 & 11.75 & -21.6 & 1.07 & 3.74 \\
\hline $\mathbf{R b}$ & ............. & NGC 3681 & 12.20 & -19.5 & 1.20 & 3.72 \\
\hline $\mathbf{R f}$ & $\ldots \ldots$ & NGC 1156 & 11.92 & -18.3 & 6.45 & 15.31 \\
\hline $\mathbf{R f}$ & & NGC 1569 & 10.58 & -16.7 & 57.19 & 60.78 \\
\hline $\mathbf{R f}$ & & NGC 3738 & 11.77 & -17.2 & 2.04 & 3.31 \\
\hline $\mathrm{Rf}$ & $\ldots \ldots$ & NGC 4214 & 9.91 & -18.9 & 17.84 & 29.93 \\
\hline $\mathbf{R f}$ & $\ldots \ldots$ & IC 5152 & 11.44 & -14.6 & 3.70 & 8.82 \\
\hline $\mathbf{R f}$ & & NGC 3664 & 12.57 & -19.4 & 0.99 & 1.84 \\
\hline Rf & & NGC 3782 & 12.87 & -18.1 & 1.46 & 3.42 \\
\hline $\mathbf{R f}$ & & NGC 4190 & 12.84 & -15.5 & 0.40 & 1.49 \\
\hline Rf & & NGC 4517A & 12.25 & -19.9 & 0.35 & 1.13 \\
\hline $\mathbf{R f}$ & & NGC 4656 & 10.00 & -20.5 & 7.36 & 12.53 \\
\hline $\mathbf{R f}$ & $\ldots$. & NGC 4861 & 12.24 & -18.8 & 2.26 & 2.94 \\
\hline Rf & ........ & NGC 5464 & 12.81 & -20.6 & 2.00 & 3.21 \\
\hline
\end{tabular}

were detected with signals well above 4 times the rms noise $(\sigma)$ and with MISS smaller than 0.5 in both bands. MISS is an estimate of the angular deviation of the IRAS signal peak from the central position used for co-adding, i.e., from the optical position of the galaxy. In order to correct for the large angular size of some of the galaxies, we used an "extended" estimate of the total flux density (in janskys) for their signal. This was obtained from the Addscan routine by integration within the signal range defined to be \pm 2.5 and \pm 4.0 from the central optical position at 60 and $100 \mu \mathrm{m}$, respectively. For small galaxies the peak flux, or maximum reading (in janskys) within the signal range, can be used for estimating the $f_{60}$ and $f_{100}$ fluxes. A plot of the difference $\Delta f$ between the flux obtained from reading the peak and the flux obtained from the "extended" estimate shows that the peak is a good estimate for sources with apparent blue magnitude $B_{T}^{0}>11.8$. We therefore use the extended estimate for galaxies with $B_{T}^{0} \leq 11.8$ if Addscan data were requested and available; if not, for all galaxies with $B_{T}^{0} \leq$ 11.8 we use a correction $\Delta f_{60}=\exp \left[-1.6\left(B_{T}^{0}-11.14\right)\right]-0.35$ at $60 \mu \mathrm{m}$ and $\Delta f_{100}=\exp \left[-1.7\left(B_{T}^{0}-11.08\right)\right]-0.29$ at 100 $\mu \mathrm{m}$ (as the best fit to $\Delta f$ data points suggests). For the close pair of galaxies NGC 3395 and NGC 3396 the two Addscan 
outputs centered at the two separate optical locations give essentially identical results, i.e., IRAS could not separate the FIR emission from the pair. We therefore assigned half of the FIR signal to each of the two galaxies.

The Vo sample consists of galaxies chosen from the " normal galaxy" list of Helou et al. (1988) in the Virgo Cluster. We selected only spiral galaxies of type $\mathrm{Sb}$ or later, but $\mathrm{S}$ ? of $\mathrm{S}$ pec galaxies are included. In order to make an appropriate correction to the blue magnitude for internal absorption, we restricted our selection to only galaxies listed in the VCC (Binggeli, Sandage, \& Tammann 1985) or in the RC2. The uncorrected blue magnitudes were taken from de Vaucouleurs \& Pence (1979), and corrections were applied using RC2 data. If no data appeared in the RC2, data from the VCC were used in order to evaluate the internal absorption, Galactic extinction, and redshift corrections for the blue magnitude. One galaxy, NGC 7279, was undetected at $60 \mu \mathrm{m}$, and another one, NGC 7326, was undetected at $100 \mu \mathrm{m}$. Considering all the other galaxies which have a positive detection both at $60 \mu \mathrm{m}$ and at $100 \mu \mathrm{m}$, as listed in Table 1 of Helou et al. (1988), we end up with a total of 80 galaxies in our Vo sample. The distance to the Virgo Cluster is evaluated using a Hubble constant of 75 $\mathrm{km} \mathrm{s}^{-1} \mathrm{Mpc}^{-1}$ and a velocity of $1300 \mathrm{~km} \mathrm{~s}^{-1}$, after correction for Virgo infall.

\subsection{Samples in the A400 and Cancer Clusters}

Optical data for the Abell 400 and Cancer clusters were obtained using the Minnesota Automated Plate Scanner, with scanning technique and method for discrimination between stars and galaxies as described by Dickey et al. (1987). We estimate the completeness of our catalog to be about $95 \%$ at $16.5 \mathrm{mag}, 90 \%$ at $17.5 \mathrm{mag}$, and $75 \%$ at $18.5 \mathrm{mag}$, by comparison with the catalogs of Butcher \& Oemler (1985) and Bothun et al. (1983). Although the scanner measures image center positions accurately, photometric calibration is difficult to obtain. For our catalog we estimate magnitudes roughly from the areas at different plate densities by comparison with the photometry of Butcher \& Oemler (1985) (who give $J$ and $F$ magnitudes for A400 galaxies) or Bothun et al. (1983) (who give $B$ and $R$ magnitudes for Cancer galaxies), so unfortunately there is a scale change in our colors between the two clusters. When it is necessary to convert between systems, we use the simple average values: $m_{B} \simeq m_{J}+0.7, B-R \simeq(J-F)+0.86$.

For most galaxies we do not have morphological types, and in order to separate spirals we have to use the optical color as an indicator. Comparing with classifications by Dressler (1980) for A400 and by Nilson (1973) for Cancer galaxies, we find that the restriction $J-F \leq 1.1$ for A400 galaxies and $B-R \leq 2.0$ for Cancer galaxies corresponds very roughly to selecting Hubble types later than Sa. This restriction in fact eliminates about $90 \%$ of the brighter galaxies in A400 which are classified as elliptical or S0 by Dressler (1980) and about $70 \%$ of the galaxies in the Cancer Cluster classified as ellipticals or S0 by Nilson (1973). Using these considerations, we divided the brighter galaxies in the A400 and Cancer clusters into four subgroups or classes: Ab: A400-bright, $m_{J}<15.5$ and $J-F \leq 1.10$ (30 galaxies); Af: A400-faint, $15.5 \leq m_{J}<16.5$ and $J-F \leq 1.10$ (49 galaxies); Cb: Cancer-bright, $m_{B}<15.5$ and $B-R \leq 2.0$ (18 galaxies); Cf: Cancer-faint, $15.5 \leq m_{B}<$ 17.0 and $B-R \leq 2.0$ (34 galaxies). About $50 \%$ of the galaxies in the MAPS catalog for A400 are redder than $J-F=1.1$, and about $20 \%$ of the galaxies in the MAPS catalog for Cancer are redder than $B-R=2.0$. None of those appear in the subsets defined above (we also obtained Addscan data for some redder and/or fainter galaxies, but, as expected, the detection fraction was small).

We have obtained IRAS data for all the galaxies in these groups using the Addscan routine. Because confusion by nearby sources is frequently a problem, we choose a rather stringent upper limit on allowed values of MISS (the difference between the FIR source position and the optical galaxy center). We consider an Addscan output to be a detection only if the signal is above 3.5 (4.0) $\sigma$ and if MISS (in absolute value) is less than 0.65 (1.5) for the $60(100) \mu \mathrm{m}$ bands, respectively. The detection rate at $60(100) \mu \mathrm{m}$ was about $60 \%(20 \%), 20 \%$ $(10 \%), 65 \%(65 \%)$, and $35 \%(30 \%)$ for the subgroups $\mathrm{Ab}$, Af, $\mathrm{Cb}$, and $\mathrm{Cf}$, respectively. The list of galaxies for the subgroups $\mathrm{Ab}, \mathrm{Af}, \mathrm{Cb}$, and $\mathrm{Cf}$ is presented in Table 2. The first three columns of Table 2 give an identifying name and the position. The third column gives the "corrected" blue magnitude, $m_{B}^{0}=$ $m_{B}-0.24-0.31$ for galaxies in Cancer and $m_{B}^{0}=m_{B}-0.24$ -0.32 for galaxies in $\mathrm{A} 400$. The subtracted 0.24 is an average correction for internal absorption, and $0.31(0.32)$ is for the Galactic extinction for Cancer (A400). Errors for these magnitudes are of the order of $\pm 0.3( \pm 0.4)$. (For galaxies in Cancer, $m_{B}$ is the blue magnitude as given by the MAPS; it is replaced by $m_{J}+0.7$ for galaxies in A400.) The fourth column gives the (uncorrected) color index $B-R(B-R \simeq J-F+0.86$ is used for galaxies in the $\mathrm{A} 400$ cluster). The following columns give the flux and the rms error in janskys, $\sigma$, and the MISS at 60 and $100 \mu \mathrm{m}$ from the Addscan routine described above. The notation " 1 " in column (12) of Table 2 indicates that we accept the source as a detection at $60 \mu \mathrm{m}$, and equivalently for $100 \mu \mathrm{m}$ in column (13).

The notation " 0 " in column (12) (col. [13]) of Table 2 indicates galaxies which have a nearby confusing signal at 60 (100) $\mu \mathrm{m}$ in the scan. The notation " 2 " in the same columns indicates the undetected galaxies. We will see that for a discussion of the $60 \mu \mathrm{m}$ distribution function for A400 and the Cancer Cluster $(\S 5)$, both detected and undetected galaxies are important. In the sample at $60 \mu \mathrm{m}$ we will exclude galaxies which have a zero in column (12) of Table 2 which do not clearly belong to the detected our undetected category. This in practice eliminates all the Addscan traces with a positive or negative signal exceeding $3.5 \sigma$ between 0.65 and 3.0 from the optical center position of the galaxy.

\section{THE JOINT FIR-OPTICAL LUMINOSITY DISTRIBUTION}

Using our samples $\mathrm{Rb}$, Rf, and Vo which go very faint in FIR and optical $L_{B}$, we have data for the joint FIR-optical luminosity function for normal late-type galaxies. Let $\chi_{B}\left(L_{B}\right)=\left(\log _{10} e\right) L_{B} \Phi\left(L_{B}\right)$ be the optical luminosity function per (decimal) logarithmic interval for late-type galaxies, irrespective of FIR properties, when $\Phi d L_{B}$ is the number per unit volume between $L_{B}$ and $L_{B}+d L_{B}$. Instead of considering $L_{B}$ and the far-infrared luminosity $L_{F I R}$ as independent variables, we chose $L_{B}$ and the distance-invariant ratio

$$
r \equiv L_{\mathrm{FIR}} / L_{\mathrm{B}}
$$

The number of galaxies per logarithmic interval of $L_{B}$ and $r$ is then given by $\chi_{B}\left(L_{B}\right)$ times the bivariate distribution function $\Psi\left(L_{B}, r\right)$, normalized so that

$$
\int_{-\infty}^{+\infty} \Psi\left(L_{B}, r\right) d\left(\log _{10} r\right)=1 .
$$


TABLE 2

Data for Ab, Af, $\mathrm{Cb}$, Cf Samples

\begin{tabular}{|c|c|c|c|c|c|c|c|c|c|c|c|c|}
\hline $\begin{array}{c}\text { name } \\
(1)\end{array}$ & $\begin{array}{l}\text { R.A. } \\
\text { (2) }\end{array}$ & $\begin{array}{c}\text { DEC } \\
(3)\end{array}$ & $\begin{array}{l}m_{B}^{0} \\
(4)\end{array}$ & $\begin{array}{c}B-R \\
(5)\end{array}$ & $\begin{array}{l}\sigma_{60} \\
(6)\end{array}$ & $\begin{array}{l}f_{60} \\
(7)\end{array}$ & $\begin{array}{l}\text { MISS }_{60} \\
(8)\end{array}$ & $\begin{array}{l}\sigma_{100} \\
(9)\end{array}$ & $\begin{array}{l}f_{100} \\
(10)\end{array}$ & $\begin{array}{l}\text { MISS }_{100} \\
(11)\end{array}$ & \multicolumn{2}{|c|}{ (12) (13) } \\
\hline$A b 1$ & 25035.9 & 54930 & 15.63 & 1.84 & 0.041 & 0.20 & 0.07 & 0.80 & 0.00 & 0.00 & 1 & 2 \\
\hline$A b 2$ & 25059.0 & 55127 & 15.15 & 1.86 & 0.037 & 0.47 & 0.03 & 0.45 & 0.00 & 0.00 & 1 & 2 \\
\hline$A b 3$ & 25120.2 & 54707 & 14.53 & 1.72 & 0.042 & 0.44 & 0.02 & 0.85 & 0.00 & 1.13 & 1 & 2 \\
\hline$A b 4$ & 25156.6 & 50256 & $15: 50$ & 1.94 & 0.033 & 0.86 & 0.03 & 0.19 & 1.75 & 0.17 & 1 & 1 \\
\hline$A b 5$ & 25241.3 & 55521 & 14.61 & 1.90 & 0.057 & 0.23 & 0.37 & 0.39 & 0.82 & 3.60 & 1 & 2 \\
\hline$A b 6$ & 25308.3 & 60056 & 13.91 & 1.92 & 0.056 & 0.26 & 0.14 & 0.26 & 1.20 & 0.14 & 1 & 1 \\
\hline$A b 7$ & 25318.7 & 61735 & 13.75 & 1.88 & 0.042 & 0.30 & 0.18 & 0.58 & 0.00 & 0.69 & 1 & 2 \\
\hline$A b 8$ & 25344.0 & 55714 & 14.79 & 1.82 & 0.046 & 0.28 & 0.12 & 0.61 & 0.00 & 0.00 & 1 & 2 \\
\hline$A b 9$ & 25407.2 & 55112 & 15.17 & 1.72 & 0.072 & 0.28 & 0.14 & 1.01 & 0.00 & 0.00 & 1 & 2 \\
\hline$A b 10$ & 25418.0 & 60018 & 14.91 & 1.94 & 0.047 & 0.18 & 0.04 & 0.32 & 2.67 & 0.29 & 1 & 1 \\
\hline$A b 11$ & 25431.2 & 50714 & 13.97 & 1.93 & 0.045 & 0.14 & 0.25 & 0.42 & 0.90 & 0.63 & 2 & 2 \\
\hline$A b 12$ & 25450.9 & 50120 & 14.89 & 1.71 & 0.042 & 0.15 & 0.08 & 0.23 & 1.34 & 0.74 & 1 & 1 \\
\hline$A b 13$ & 25452.7 & 75356 & 14.56 & 1.82 & 0.030 & 0.25 & 0.05 & 0.36 & 0.84 & 0.48 & 1 & 2 \\
\hline$A b 14$ & 25502.2 & 63545 & 15.19 & 1.88 & 0.056 & 0.14 & 0.10 & 0.20 & 2.40 & 1.96 & 2 & 0 \\
\hline$A b 15$ & 25505.4 & 62224 & 15.62 & 1.68 & 0.041 & 0.12 & 1.28 & 0.35 & 0.00 & 0.00 & 2 & 2 \\
\hline$A b 16$ & 25507.3 & 54507 & 14.67 & 1.81 & 0.056 & 0.19 & 0.13 & 0.25 & 0.61 & 0.10 & 2 & 2 \\
\hline$A b 17$ & 25519.1 & 50502 & 15.35 & 1.79 & 0.052 & 0.44 & 0.02 & 0.61 & 0.00 & 0.00 & 1 & 2 \\
\hline$A b 18$ & 25539.2 & 65449 & 15.42 & 1.91 & 0.036 & 0.23 & 0.19 & 0.55 & 1.88 & 1.76 & 1 & 2 \\
\hline$A b 19$ & 25550.8 & 60624 & 14.17 & 1.88 & 0.046 & 0.27 & 0.09 & 0.58 & 4.41 & 0.09 & 1 & 1 \\
\hline$A b 20$ & 25605.6 & 61326 & 15.54 & 1.94 & 0.023 & 0.23 & 0.00 & 0.89 & 1.92 & 1.95 & 1 & 2 \\
\hline$A b 21$ & 25607.8 & 50659 & 15.49 & 1.56 & 0.036 & 0.00 & 0.00 & 0.17 & 0.00 & 0.00 & 2 & 2 \\
\hline$A b 22$ & 25614.8 & 55732 & 15.46 & 1.85 & 0.058 & 0.00 & 0.00 & 0.34 & 0.00 & 0.00 & 2 & 2 \\
\hline$A b 23$ & 25637.4 & 55605 & 14.72 & 1.85 & 0.017 & 0.45 & 0.08 & 0.14 & 1.48 & 0.33 & 1 & 1 \\
\hline$A b 24$ & 25641.3 & 50514 & 14.77 & 1.76 & 0.050 & 0.13 & 1.45 & 0.18 & 0.48 & 0.01 & 2 & 2 \\
\hline$A b 25$ & 25713.3 & 62005 & 14.52 & 1.91 & 0.073 & 0.00 & 0.00 & 0.85 & 0.00 & 0.00 & 2 & 2 \\
\hline$A b 26$ & 25721.4 & 54610 & 14.90 & 1.84 & 0.025 & 0.15 & 0.14 & 0.24 & 0.00 & 0.00 & 1 & 2 \\
\hline$A b 27$ & 25730.2 & 53623 & 15.23 & 1.91 & 0.056 & 0.12 & 0.61 & 0.36 & 0.00 & 0.00 & 2 & 2 \\
\hline$A b 28$ & 25731.3 & 53049 & 15.15 & 1.91 & 0.050 & 0.00 & 0.00 & 0.17 & 0.48 & 3.79 & 2 & 2 \\
\hline$A b 29$ & 25913.9 & 53116 & 15.54 & 1.27 & 0.028 & 0.08 & 0.90 & 0.23 & 0.00 & 0.00 & 2 & 2 \\
\hline$A b 30$ & 30200.9 & 51456 & 14.59 & 1.88 & 0.033 & 0.00 & 0.00 & 0.31 & 0.00 & 0.25 & 2 & 2 \\
\hline Af 1 & 24832.5 & 75230 & 16.52 & -0.21 & 0.038 & 0.09 & 0.16 & 0.37 & 0.00 & 0.00 & 0 & 0 \\
\hline Af 2 & 24843.1 & 52102 & 16.48 & 1.85 & 0.060 & 0.00 & 0.00 & 0.33 & 0.00 & 0.00 & 2 & 0 \\
\hline Af 3 & 24913.9 & 55054 & 16.14 & 1.87 & 0.107 & 0.32 & 2.15 & 0.77 & 2.99 & 1.65 & 2 & 2 \\
\hline Af 4 & 24959.5 & 50543 & 16.24 & 1.80 & 0.021 & 0.15 & 0.10 & 0.47 & 1.15 & 1.42 & 1 & 2 \\
\hline Af 5 & 25011.0 & 64223 & 16.02 & 1.84 & 0.034 & 0.15 & 0.24 & 0.33 & 1.17 & 0.39 & 1 & 2 \\
\hline Af 6 & 25109.7 & 62749 & 16.08 & 1.81 & 0.041 & 0.11 & 0.33 & 0.32 & 0.00 & 0.00 & 2 & 2 \\
\hline Af 7 & 25130.5 & 51054 & 16.46 & 1.73 & 0.040 & 0.13 & 0.13 & 0.21 & 0.94 & 0.58 & 2 & 1 \\
\hline Af 8 & 25146.8 & 54845 & 16.30 & 1.71 & 0.048 & 0.00 & 0.00 & 0.90 & 0.00 & 0.00 & 2 & 2 \\
\hline Af 9 & 25149.0 & 51054 & 16.07 & 1.96 & 0.039 & 0.00 & 0.00 & 0.43 & 0.00 & 0.00 & 2 & 2 \\
\hline Af 10 & 25210.6 & 61147 & 16.48 & 1.87 & 0.048 & 0.00 & 0.00 & 0.35 & 0.00 & 0.00 & 2 & 2 \\
\hline Af 11 & 25223.3 & 52432 & 15.81 & 1.89 & 0.043 & 0.00 & 0.00 & 0.32 & 0.00 & 0.00 & 2 & 2 \\
\hline Af 12 & 25312.9 & 71524 & 15.83 & 1.68 & 0.057 & 0.20 & 0.77 & 0.38 & 1.05 & 0.44 & 0 & 2 \\
\hline Af 13 & 25324.3 & 74942 & 16.10 & 1.96 & 0.065 & 0.00 & 0.00 & 0.35 & 1.86 & 2.50 & 2 & 0 \\
\hline Af 14 & 25328.6 & 75317 & 16.45 & 1.96 & 0.048 & 0.17 & 0.02 & 0.57 & 0.00 & 0.00 & 1 & 2 \\
\hline Af 15 & 25334.1 & 55444 & 15.83 & 1.74 & 0.052 & 0.00 & 0.00 & 0.43 & 0.00 & 0.00 & 2 & 2 \\
\hline Af 16 & 25341.8 & 73215 & 16.50 & 1.89 & 0.043 & 0.24 & 1.96 & 0.47 & 1.05 & 1.34 & 0 & 2 \\
\hline Af 17 & 25347.0 & 60750 & 16.51 & 1.83 & 0.040 & 0.12 & 0.84 & 0.35 & 0.00 & 0.00 & 2 & 2 \\
\hline Af 18 & 25353.0 & 60443 & 16.62 & 1.95 & 0.050 & 0.00 & 0.00 & 0.59 & 0.00 & 0.00 & 2 & 2 \\
\hline Af 19 & 25406.4 & 52918 & 16.35 & 1.89 & 0.030 & 0.14 & 0.07 & 0.27 & 0.00 & 0.00 & 1 & 2 \\
\hline Af 20 & 25420.8 & 61958 & 16.10 & 1.85 & 0.058 & 0.12 & 2.30 & 0.34 & 1.96 & 2.42 & 2 & 0 \\
\hline$A f 21$ & 25453.6 & 73834 & 16.41 & 1.93 & 0.081 & 0.23 & 0.13 & 0.68 & 1.43 & 0.11 & 2 & 2 \\
\hline Af 22 & 25457.7 & 52819 & 16.61 & 1.62 & 0.025 & 0.00 & 0.00 & 0.22 & 0.00 & 0.00 & 2 & 0 \\
\hline Af 23 & 25459.0 & 61058 & 15.80 & 1.92 & 0.025 & 0.11 & 0.09 & 0.17 & 0.00 & 0.00 & 1 & 2 \\
\hline$A f 24$ & 25520.1 & 65229 & 16.53 & 1.77 & 0.040 & 0.23 & 0.06 & 0.61 & 0.00 & 0.00 & 1 & 2 \\
\hline Af 25 & 25521.6 & 54332 & 15.98 & 1.64 & 0.054 & 0.24 & 1.07 & 0.32 & 0.91 & 0.69 & 0 & 2 \\
\hline Af 26 & 25524.2 & 54524 & 16.25 & 1.42 & 0.027 & 0.26 & 0.55 & 0.24 & 1.07 & 1.37 & 1 & 1 \\
\hline Af 27 & 25610.1 & 52426 & 15.82 & 1.89 & 0.037 & 0.13 & 0.32 & 0.34 & 0.77 & 2.18 & 2 & 2 \\
\hline Af 28 & 25617.1 & 60515 & 15.83 & 1.91 & 0.071 & 0.00 & 0.00 & 0.25 & 2.11 & 0.51 & 2 & 1 \\
\hline Af 29 & 25646.7 & 55001 & 16.50 & 1.58 & 0.037 & 0.00 & 0.00 & 0.17 & 0.53 & 0.61 & 2 & 0 \\
\hline Af 30 & 25653.2 & 52608 & 16.47 & 1.75 & 0.040 & 0.00 & 0.00 & 0.19 & 0.00 & 0.47 & 2 & 2 \\
\hline Af 31 & 25655.5 & 50808 & 15.65 & 1.72 & 0.031 & 0.07 & 0.03 & 0.12 & 0.54 & 2.32 & 2 & 0 \\
\hline Af 32 & 25656.4 & 70434 & 15.77 & 1.91 & 0.040 & 0.00 & 0.00 & 0.65 & 0.00 & 0.00 & 2 & 2 \\
\hline Af 33 & 25703.2 & 51325 & 15.69 & 1.79 & 0.034 & 0.00 & 0.00 & 0.17 & 0.00 & 0.00 & 2 & 0 \\
\hline Af 34 & 25704.3 & 53342 & 15.71 & 1.96 & 0.027 & 0.00 & 0.00 & 0.10 & 0.00 & 0.00 & 0 & 2 \\
\hline Af 35 & 25710.5 & 54738 & 16.38 & 1.74 & 0.048 & 0.00 & 0.00 & 0.40 & 0.00 & 0.00 & 0 & 2 \\
\hline Af 36 & 25714.8 & 50602 & 16.08 & 1.57 & 0.036 & 0.13 & 0.64 & 0.15 & 0.57 & 1.08 & 1 & 2 \\
\hline Af 37 & 25714.2 & 61856 & 16.05 & 1.83 & 0.098 & 0.00 & 0.00 & 0.69 & 0.00 & 0.00 & 2 & 2 \\
\hline Af 38 & 25714.3 & 50424 & 16.44 & 1.22 & 0.026 & 0.10 & 0.00 & 0.30 & 0.82 & 0.31 & 1 & 2 \\
\hline Af 39 & 25716.1 & 53226 & 16.62 & 1.95 & 0.022 & 0.08 & 0.22 & 0.22 & 0.61 & 0.28 & 1 & 2 \\
\hline Af 40 & 25730.3 & 70749 & 15.90 & 1.80 & 0.027 & 0.07 & 2.50 & 0.63 & 0.00 & 0.00 & 2 & 2 \\
\hline
\end{tabular}


TABLE 2-Continued

\begin{tabular}{|c|c|c|c|c|c|c|c|c|c|c|c|c|}
\hline $\begin{array}{l}\text { name } \\
(1)\end{array}$ & $\begin{array}{l}\text { R.A. } \\
\text { (2) }\end{array}$ & $\begin{array}{c}\text { DEC } \\
(3)\end{array}$ & $\begin{array}{l}m_{B}^{0} \\
(4)\end{array}$ & $\begin{array}{c}B-R \\
(5)\end{array}$ & $\begin{array}{l}\sigma_{60} \\
(6)\end{array}$ & $\begin{array}{l}f_{60} \\
(7)\end{array}$ & $\begin{array}{l}\text { MISS }_{60} \\
(8)\end{array}$ & $\begin{array}{c}\sigma_{100} \\
(9)\end{array}$ & $\begin{array}{l}f_{100} \\
(10)\end{array}$ & $\begin{array}{l}\text { MISS }_{100} \\
\text { (11) }\end{array}$ & \multicolumn{2}{|c|}{ (12) (13) } \\
\hline Af 41 & 25730.4 & 51910 & 16.34 & 1.51 & 0.022 & 0.06 & 2.02 & 0.14 & 0.00 & 0.00 & 2 & 2 \\
\hline$A f 42$ & 25749.8 & 50716 & 16.02 & 0.83 & 0.034 & 0.00 & 0.00 & 0.20 & 0.00 & 0.00 & 2 & 2 \\
\hline Af 43 & 25752.2 & 54726 & 16.32 & 1.65 & 0.043 & 0.00 & 0.00 & 0.32 & 0.00 & 0.00 & 2 & 2 \\
\hline Af 44 & 25828.9 & 53312 & 16.54 & 1.91 & 0.024 & 0.10 & 1.04 & 0.12 & 0.00 & 0.16 & 0 & 2 \\
\hline$A f 45$ & 25902.2 & 51641 & 16.24 & 1.82 & 0.035 & 0.26 & 0.22 & 0.21 & 0.00 & 0.33 & 1 & 2 \\
\hline$A f 46$ & 25903.7 & 55327 & 16.42 & 1.71 & 0.041 & 0.23 & 0.93 & 0.16 & 0.00 & 0.00 & 0 & 2 \\
\hline Af 47 & 25921.8 & 74844 & 16.25 & -0.18 & 0.078 & 0.26 & 2.08 & 0.39 & 3.63 & 1.08 & 2 & 1 \\
\hline$A f 48$ & 25946.3 & 54620 & 16.38 & 1.88 & 0.027 & 0.07 & 0.47 & 0.11 & 0.00 & 0.00 & 2 & 2 \\
\hline Af 49 & 30006.8 & 54741 & 16.61 & 1.89 & 0.042 & 0.00 & 0.00 & 0.16 & 0.36 & 2.50 & 2 & 2 \\
\hline $\mathrm{Cb} 1$ & 81338.3 & 213353 & 14.65 & 1.86 & 0.032 & 0.13 & 1.58 & 0.15 & 0.00 & 0.00 & 0 & 2 \\
\hline$C b 2$ & 81429.9 & 215030 & 12.70 & 1.45 & 0.040 & 1.26 & 0.16 & 0.11 & 3.48 & 0.07 & 1 & 1 \\
\hline $\mathrm{Cb} 3$ & 81440.0 & 210331 & 14.51 & 1.94 & 0.022 & 0.17 & 2.53 & 0.09 & 0.43 & 2.53 & 0 & 0 \\
\hline $\mathrm{Cb} 4$ & 81534.8 & 205504 & 14.80 & 1.68 & 0.041 & 0.59 & 0.04 & 0.11 & 1.82 & 0.04 & 1 & 1 \\
\hline$C b 5$ & 81606.6 & 212034 & 13.78 & 1.15 & 0.041 & 0.55 & 0.14 & 0.12 & 1.67 & 0.12 & 1 & 1 \\
\hline $\mathrm{Cb} 6$ & 81609.3 & 215656 & 14.35 & 1.54 & 0.035 & 0.97 & 0.07 & 0.06 & 2.68 & 0.13 & 1 & 1 \\
\hline$C b 7$ & 81618.3 & 204005 & 13.74 & 1.71 & 0.038 & 0.18 & 0.40 & 0.18 & 1.01 & 0.57 & 1 & 1 \\
\hline $\mathrm{Cb} 8$ & 81636.8 & 213307 & 14.57 & 1.65 & 0.029 & 0.21 & 0.12 & 0.09 & 0.90 & 0.05 & 1 & 1 \\
\hline$C b 9$ & 81642.7 & 211621 & 14.69 & 1.30 & 0.037 & 0.83 & 0.02 & 0.12 & 2.36 & 0.12 & 1 & 1 \\
\hline$C b 10$ & 81722.0 & 210201 & 14.87 & 0.59 & 0.051 & 0.10 & 0.10 & 0.20 & 0.52 & 0.07 & 2 & 2 \\
\hline$C b 11$ & 81827.8 & 210412 & 14.79 & 1.19 & 0.046 & 0.25 & 0.05 & 0.10 & 0.83 & 0.23 & 1 & 1 \\
\hline$C b 12$ & 81906.2 & 213016 & 14.89 & 0.51 & 0.029 & 0.07 & 0.12 & 0.15 & 0.00 & 0.00 & 2 & 2 \\
\hline$C b 13$ & 82039.3 & 213036 & 14.51 & 1.49 & 0.034 & 1.59 & 0.37 & 0.12 & 3.14 & 0.42 & 1 & 1 \\
\hline$C b 14$ & 82046.3 & 213549 & 14.52 & 1.82 & 0.050 & 0.15 & 0.25 & 0.17 & 1.56 & 0.25 & 0 & 1 \\
\hline$C b 15$ & 82106.9 & 211121 & 13.90 & 1.95 & 0.035 & 0.69 & 0.10 & 0.09 & 2.98 & 0.12 & 1 & 1 \\
\hline$C b 16$ & 82312.6 & 213717 & 14.72 & 1.31 & 0.032 & 0.22 & 0.05 & 0.34 & 0.00 & 0.00 & 1 & 2 \\
\hline$C b 17$ & 82410.8 & 214839 & 14.27 & 1.25 & 0.059 & 0.00 & 0.00 & 0.14 & 0.00 & 0.00 & 2 & 2 \\
\hline$C b 18$ & 82447.1 & 213838 & 12.79 & 1.44 & 0.033 & 0.94 & 0.08 & 0.13 & 3.46 & 0.06 & 1 & 1 \\
\hline$C f 1$ & 81230.0 & 214300 & 15.15 & 0.00 & 0.038 & 0.09 & 0.51 & 0.14 & 0.00 & 0.00 & 2 & 2 \\
\hline Cf 2 & 81248.5 & 211707 & 15.92 & 0.60 & 0.042 & 0.11 & 0.28 & 0.11 & 0.45 & 1.59 & 2 & 0 \\
\hline$C f 3$ & 81257.7 & 203923 & 16.01 & 1.60 & 0.030 & 0.47 & 0.14 & 0.11 & 1.22 & 0.92 & 1 & 1 \\
\hline$C f 4$ & 81403.0 & 204003 & 15.16 & 1.55 & 0.031 & 0.10 & 0.27 & 0.11 & 0.36 & 0.96 & 2 & 2 \\
\hline$C f 5$ & 81531.0 & 205639 & 15.34 & 1.51 & 0.054 & 0.40 & 1.11 & 0.12 & 1.82 & 1.32 & 0 & 1 \\
\hline$C f 6$ & 81553.9 & 212231 & 15.13 & 1.61 & 0.030 & 0.16 & 0.39 & 0.11 & 0.80 & 0.71 & 1 & 1 \\
\hline$C f 7$ & 81605.9 & 210540 & 15.57 & 1.88 & 0.044 & 0.17 & 0.02 & 0.33 & 0.00 & 0.00 & 1 & 2 \\
\hline Cf 8 & 81612.3 & 220014 & 16.25 & 1.86 & 0.046 & 0.26 & 1.12 & 0.10 & 2.64 & 3.61 & 0 & 0 \\
\hline$C f 9$ & 81618.3 & 205454 & 15.13 & 0.80 & 0.036 & 0.14 & 2.17 & 0.15 & 0.53 & 0.06 & 0 & 2 \\
\hline$C f 10$ & 81624.8 & 211258 & 15.11 & 1.29 & 0.047 & 0.22 & 0.01 & 0.20 & 0.62 & 0.44 & 1 & 2 \\
\hline$C f 11$ & 81642.8 & 212635 & 16.24 & 1.73 & 0.049 & 0.00 & 0.00 & 0.15 & 0.44 & 2.85 & 2 & 2 \\
\hline$C f 12$ & 81703.9 & 211327 & 15.59 & 1.41 & 0.048 & 0.22 & 0.05 & 0.08 & 0.77 & 0.79 & 1 & 1 \\
\hline$C f 13$ & 81715.8 & 211339 & 15.22 & 1.95 & 0.048 & 0.00 & 0.00 & 0.19 & 0.00 & 0.00 & 2 & 2 \\
\hline$C f 14$ & 81736.4 & 215244 & 15.87 & 0.68 & 0.037 & 0.09 & 0.31 & 0.11 & 0.00 & 0.00 & 2 & 2 \\
\hline$C f 15$ & 81820.6 & 213746 & 16.03 & 1.26 & 0.027 & 0.00 & 0.00 & 0.14 & 0.00 & 0.00 & 2 & 2 \\
\hline$C f 16$ & 81823.7 & 213623 & 16.22 & 1.91 & 0.031 & 0.09 & 0.39 & 0.06 & 0.32 & 1.79 & 2 & 0 \\
\hline$C f 17$ & 81836.0 & 211700 & 15.15 & 0.00 & 0.040 & 0.00 & 0.00 & 0.17 & 0.39 & 2.44 & 2 & 2 \\
\hline$C f 18$ & 81906.3 & 213010 & 14.99 & 1.61 & 0.029 & 0.07 & 0.03 & 0.15 & 0.00 & 0.00 & 2 & 2 \\
\hline$C f 19$ & 81941.2 & 210926 & 15.98 & 1.78 & 0.055 & 0.00 & 0.00 & 0.16 & 0.00 & 0.00 & 2 & 2 \\
\hline$C f 20$ & 81958.2 & 211421 & 15.49 & 1.91 & 0.037 & 0.11 & 0.68 & 0.16 & 0.49 & 2.95 & 0 & 2 \\
\hline$C f 21$ & 82037.5 & 212959 & 15.13 & 1.47 & 0.037 & 1.51 & 0.32 & 0.15 & 3.13 & 0.28 & 1 & 1 \\
\hline$C f 22$ & 82057.2 & 213450 & 16.27 & 1.53 & 0.075 & 0.17 & 0.28 & 0.31 & 0.00 & 0.00 & 2 & 2 \\
\hline$C f 23$ & 82100.6 & 210815 & 15.12 & 1.95 & 0.037 & 0.13 & 0.01 & 0.11 & 2.86 & 3.27 & 0 & 0 \\
\hline$C f 24$ & 82126.1 & 204145 & 15.47 & 1.55 & 0.029 & 0.12 & 0.04 & 0.08 & 0.46 & 0.07 & 1 & 1 \\
\hline$C f 25$ & 82134.4 & 211226 & 15.82 & 1.41 & 0.025 & 0.21 & 0.27 & 0.14 & 0.44 & 1.12 & 1 & 2 \\
\hline$C f 26$ & 82157.4 & 210916 & 16.25 & 1.93 & 0.031 & 0.11 & 0.51 & 0.16 & 0.40 & 1.06 & 1 & 2 \\
\hline$C f 27$ & 82310.4 & 214957 & 15.67 & 1.30 & 0.041 & 0.21 & 2.18 & 0.16 & 0.00 & 0.00 & 0 & 2 \\
\hline$C f 28$ & 82324.3 & 204940 & 16.19 & 1.68 & 0.035 & 0.15 & 0.22 & 0.14 & 0.68 & 0.28 & 1 & 1 \\
\hline$C f 29$ & 82332.3 & 205746 & 15.24 & -0.08 & 0.045 & 0.18 & 0.54 & 0.18 & 0.00 & 0.00 & 1 & 2 \\
\hline$C f 30$ & 82355.2 & 203147 & 15.54 & 1.14 & 0.323 & 0.34 & 0.33 & 0.09 & 1.87 & 0.38 & 0 & 1 \\
\hline$C f 31$ & 82425.0 & 215309 & 16.42 & 1.82 & 0.051 & 0.21 & 0.10 & 0.14 & 0.64 & 0.62 & 1 & 1 \\
\hline$C f 32$ & 82441.5 & 210148 & 14.97 & 1.68 & 0.039 & 0.00 & 0.00 & 0.09 & 0.00 & 0.00 & 2 & 0 \\
\hline$C f 33$ & 82512.8 & 212211 & 16.43 & 1.20 & 0.030 & 0.09 & 0.93 & 0.12 & 0.36 & 0.93 & 2 & 2 \\
\hline$C f 34$ & 82608.8 & 210307 & 15.89 & 1.44 & 0.044 & 0.10 & 0.45 & 0.10 & 0.42 & 0.28 & 2 & 1 \\
\hline
\end{tabular}

This normalization is convenient, since samples for determining $\Psi$ can be chosen from different sky areas for different ranges of $L_{B}$, as we do with galaxies in the $\mathrm{Rb}$ and $\mathrm{Rf}$ samples. The FIR luminosity function per logarithmic interval, $\chi_{\mathrm{FIR}}\left(L_{\mathrm{FIR}}\right)=\left(\log _{10} e\right) L_{\mathrm{FIR}} \Phi_{\mathrm{FIR}}\left(L_{\mathrm{FIR}}\right)$ is then given by

$$
\chi_{\mathrm{FIR}}\left(L_{\mathrm{FIR}}\right)=\int_{-\infty}^{+\infty} \Psi\left(L_{B}, r\right) \chi_{B}\left(L_{B}\right) d\left(\log _{10} L_{B}\right),
$$

where $\chi_{\text {FIR }}$, as well as $\Psi$ and $\chi_{B}$, refers only to late-type galaxies.

Following Bothun et al. (1989) and Helou et al. (1988), we define a measure FIR for the received far-infrared flux by

$$
F_{\mathrm{FIR}}=F_{60}+F_{100}=1.26 \times 10^{-14}\left(2.58 f_{60}+f_{100}\right)
$$

in $\mathrm{W} \mathrm{m} \mathrm{m}^{-2}$, where $f_{60}$ and $f_{100}$ are in janskys. Note that this 
expression for $F_{\text {FIR }}$ does not include the color-dependent multiplying correction factor (typically between 1.3 and 1.8) for $\lambda \gg 100 \mu \mathrm{m}$ emission (see Table 3 of Helou et al. 1988) to get bolometric FIR luminosities. We also define a nominal blue flux $F_{B}=v f_{v}$, at $\lambda=4400 \AA$ assuming a calibration of $4300 \mathrm{Jy}$ for $B_{T}^{0}=0$ (Allen 1973). The explicit relation between $F_{B}$ in W $\mathrm{m}^{-2}$ and the apparent magnitude is

$$
\log _{10} F_{B}=-0.4 B_{T}^{0}-7.53
$$

As discussed in the previous section, we can evaluate, for the three samples Rb, Rf, and Vo, the absolute magnitude $M_{B}^{0}$ and the ratio $r$. The scatter diagram for $\log _{10} r$ and $M_{B}^{0}$ in Figure 1 shows that the distribution of $\log _{10} r$ depends at best only weakly on $M_{B}^{0}$, i.e., that $r$ and $L_{B}$ are not strongly correlated. If a weak correlation exists, this is due to the less luminous galaxies. In fact, the straight line in Figure 1, which is the leastsquares fit to all the data points, has a small, negative slope, i.e., $\left\langle\log _{10} r\right\rangle \propto\left(\log _{10} L_{B}\right)^{\delta}$ with $\delta=+0.08 \pm 0.04$. This nonzero value of $\delta$ is due to the presence of the low-luminosity $\mathrm{Rf}$ sample; in fact, if we omit the faintest three points at $M_{B}^{0}>$ -15.5 , then $\delta \simeq-0.03 \pm 0.03$. If dwarf galaxies are unimportant, $\delta$ can be replaced by zero and the bivariate distribution function $\Psi$ can be replaced by a one-dimensional function $\psi(r)$. If faint galaxies are to be included, we suggest a slight generalization which allows for a weak dependence of $\psi$ on $L_{B}$ : disregarding possible variations of the width of the $\log _{10} r$ distribution with $L_{B}$, we consider $\psi$ as a function of one new variable $r^{\prime}$ :

$$
\Psi\left(L_{B}, r\right)=\psi\left(r^{\prime}\right), \quad r^{\prime} \equiv r\left(L_{B} / L_{*}\right)^{-\delta}=L_{\mathrm{FIR}} L_{B}^{-(1+\delta)} L_{*}^{\delta},
$$

where $L_{*}$ is a typical luminosity for the galaxy sample. Since our sample is biased toward faint galaxies, having selected late-type spirals and having mainly faint galaxies in the $\mathbf{R f}$ group, we use $L_{*} \simeq 10^{36} \mathrm{~W}$ (corresponding to $M_{B}^{0}=-19.0$ ) and $\delta=0.08$ for numerical work. If the distance is unknown for a sample of galaxies, $r^{\prime}$ could be replaced by $r$ because $r^{\prime} / r$ varies very slowly.

The distribution of galaxies in various $r^{\prime}$ ranges is plotted in Figure 2. RSA galaxies are all together $(\mathbf{R b}+\mathbf{R f})$ in Figure $2 a$ (the hatched part indicates the sample Rf alone). In Figure $2 b$

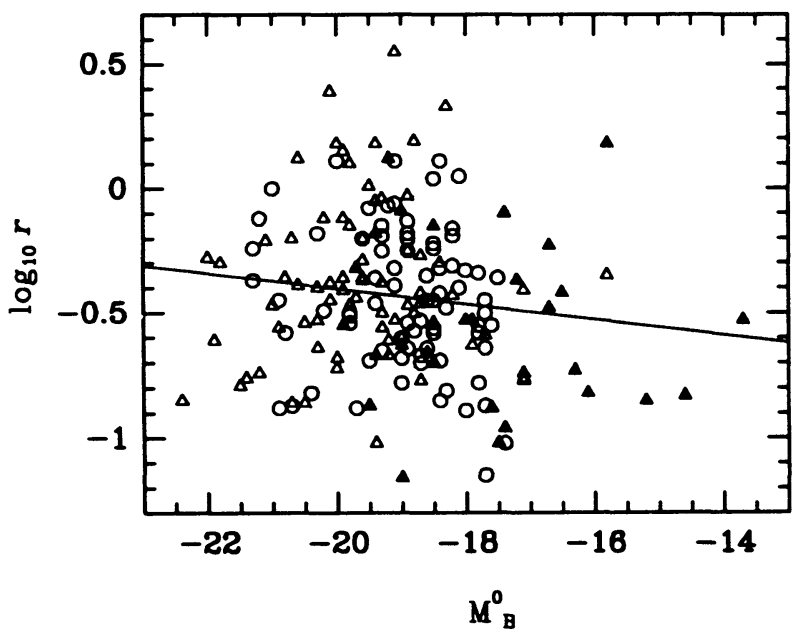

Fig. 1. - The weak correlation between $\log _{10} r=L_{\mathrm{FIR}} / L_{B}$ and $M_{B}^{0}$ is shown for all the galaxies in the $\mathbf{R b}$ sample (open triangles), in the $\mathrm{Rf}$ sample (filled triangles), and in the Vo sample (open circles). The straight line is the best fit to all the data points shown.
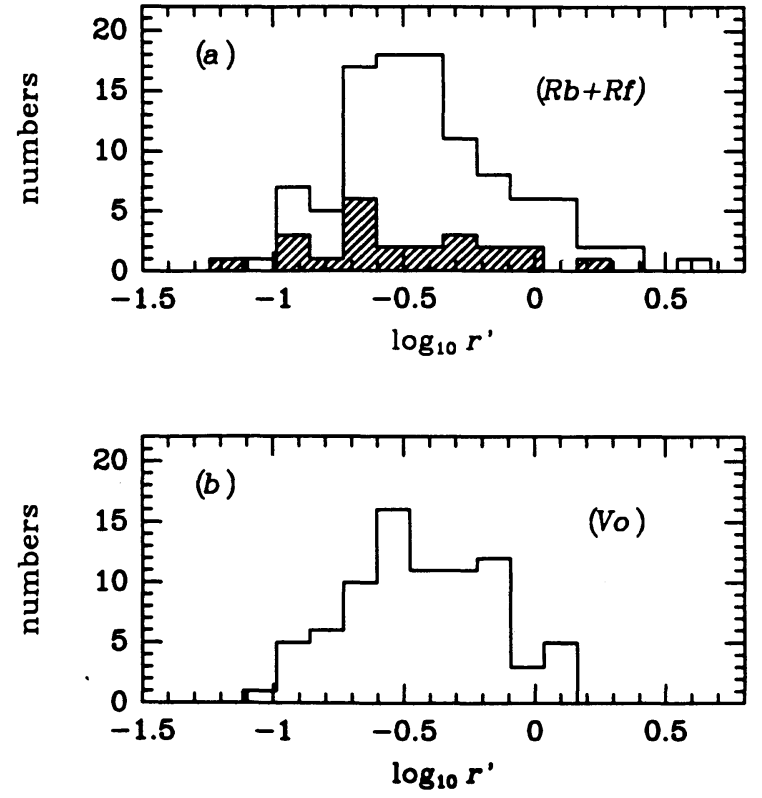

Fig. 2.-(a) Histograms of the logarithm of the ratio $r^{\prime} \equiv r\left(L_{B} / L_{*}\right)^{-\delta}$ for galaxies in the $\mathbf{R b}+\mathbf{R f}$ sample. The hatched part of the histogram is for the $\mathbf{R f}$ sample alone. (b) Histograms of the logarithm of the ratio $r^{\prime}$ for galaxies in the Vo sample.

we show the distribution over $r^{\prime}$ for galaxies in the Vo sample. The largest value of $\boldsymbol{r}^{\prime}$ for the Rf sample is 3.5 (the Sc galaxy NGC 3683), while all galaxies in Virgo have $r^{\prime}$ smaller than 1.4. The approximate one-dimensional distribution function $\psi\left(r^{\prime}\right)$ can then be determined from our data. Because the distribution in Figure $2 a$ for $\mathbf{R f}$ and $\mathbf{R b}$ galaxies is very similar to that in Figure $2 b$ for galaxies in Virgo, we can combine the samples in order to derive the analytic expression for the distribution function $\psi\left(r^{\prime}\right)$ with reasonable accuracy, expect for very large $r^{\prime}$. Figure $3 a$ shows the observational points for $r^{\prime}$ in our three samples combined. The smooth curve in Figure $3 a$ is a twoparameter fit lognormal form using the seven central data points (filled triangles). We normalize it so that its integral over $d \log _{10} r^{\prime}$ is unity,

$$
\psi\left(r^{\prime}\right)=(\gamma / \pi)^{1 / 2} \exp \left\{-\gamma\left[\log _{10}\left(r^{\prime} / r_{0}\right)\right]^{2}\right\},
$$

where the width is $\gamma=6.2 \pm 1$, the peak is at $r^{\prime}=r_{0}=0.35$, and $(\gamma / \pi)^{1 / 2}$ is the normalization factor. The dispersion of this lognormal curve is $(1 / 2 \gamma)^{1 / 2}=0.28 \pm 0.03$. All the data points lie between $r^{\prime}=0.1 r_{0}$ and $r^{\prime}=15 r_{0}$. The data points we used for the fitting are within an interval of $\log _{10} r^{\prime}$ equal to 1.3 , centered on $\log _{10} r_{0}$. In Figure $3 b$ we plot again the same data irrespective of $L_{B}$, i.e., as a function of $r$ (no $\delta$ correction); here $r_{0}=0.35$, and the width is $\gamma=5.9 \pm 1$ for the curve fitted in Figure $3 b$. Because $\delta$ is so small, and the range of $L_{B}$ is not so large, there is little difference between Figures $3 a$ and $3 b$ except perhaps for small $r$ values. Our results for $r$ agree with those of Rowan-Robinson et al. (1987), who similarly fitted their data for spiral and irregular galaxies near the peak of the curve with a lognormal curve of width $\gamma=8.7$ and peak at $r_{0}=0.3$ (the slight differences are probably due to slightly different selection criteria for Hubble type/color). However, the distribution function at the left-hand side of the peak of the lognormal curve is seen best from optically selected nearby galaxies and as a function of $r^{\prime}$. Figure $3 a$ shows an important negative result, namely, the absence of any tail above the lognormal curve for 

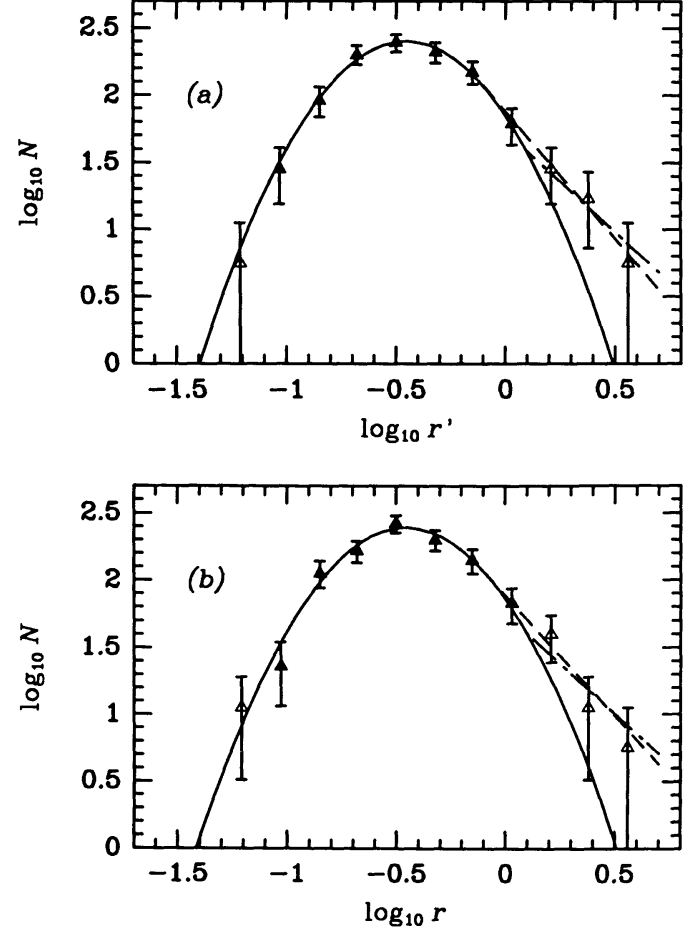

Fig. 3.-Triangles show the distribution of the logarithm of the number of galaxies $N$ in the combined $\mathrm{Rb}, \mathrm{Rf}$, and Vo sample, for intervals $(a)$ in $d \log _{10} r^{\prime}$ and $(b)$ in $d \log _{10} r$. The solid curve is a Gaussian fit to all the points marked as filled triangles. The dashed line matches the parabolic fit and its derivative at $\log _{10} r^{\prime}=-0.1$ in $(a)$ and at $\log _{10} r=-0.1$ in $(b)$, and is a good fit to the last three points (open triangles to the right). The dash-dot line has a slope of -1.5 and matches the fit at $\log _{10} r^{\prime}=0.1$ in $(a)$ and at $\log _{10} r=0.1$ in $(b)$. Error bars denote the $N^{0.5}$ uncertainties.

small $r^{\prime}$. It should be noted that not a single galaxy in our RSA sample failed to be detected in the FIR and that only two galaxies out of 82 in the original Vo sample were not detected either at 60 or at $100 \mu \mathrm{m}$.

As Rowan-Robinson et al. (1987) also showed, a tail in the distribution towards large $r$ is present in a FIR-selected sample. The tail is also visible in the optically selected sample of Bothun et al. (1989) (see their Fig. 2). The broad and symmetric lognormal curve fits our data fairly well for $r^{\prime} / r_{0} \leq 3$, but a tail shows up at large $r^{\prime} / r_{0}$, or at large $r / r_{0}$, even for our optically selected data in Figure 3 . In our data the tail comes mainly from the RSA galaxies, as expected from the histogram in Figure 2. The arithmetic average value of $r^{\prime}$ over the lognormal curve (without the tail) is $\left\langle r^{\prime}\right\rangle=r_{0} \exp \left[(\ln 10)^{2} / 4 \gamma\right]=$ 0.43 . By averaging all our observational data in samples $R b$, $\mathrm{Rf}$, Vo, we have instead a slightly larger value (due to the tail) of $\left\langle r^{\prime}\right\rangle=0.48$. The two straight lines in Figures $3 a$ and $3 b$ are good fits to the data points at large $r^{\prime}$. The distribution function $\psi$ has then at least four disposable parameters: $\delta, r_{0}, \gamma$, and the switching point, $r_{\mathrm{sw}}$. The lognormal form in equation (7) for $\psi\left(r^{\prime}\right)$ can be used for all $r^{\prime}$ less than $r_{\mathrm{sw}}^{\prime}$. We then suggest switching to a power law $\psi \propto\left(r^{\prime}\right)^{-n_{*}}$ for $r^{\prime} \geq r_{\mathrm{sw}}^{\prime}$ with a multiplying factor chosen to give continuity at $r_{\text {sw }}^{\prime}$. The dashed line is obtained by requiring the logarithmic derivative of $\psi\left(r^{\prime}\right)$ to be continuous at $\log _{10} r^{\prime}=\log _{10} r_{\text {sw }}^{\prime}=-0.1$. This condition implies that $n_{*}=(\gamma / 1.15) \log _{10}\left(r_{\mathrm{sw}}^{\prime} / r_{0}\right)=1.9$ (using always $\delta=0.08, \gamma=6.2, r_{0}=0.35$ ). If we do not require the logarithmic derivative to be continuous, we can switch to a power law at some $r^{\prime}$ beyond the last data point which is well fitted by the lognormal curve (the last filled triangle to the right in Fig. 3). The dash-dot line of slope -1.5 in Fig. $3 a$ fits the data for $\log _{10} r^{\prime}>\log _{10} r_{\text {sw }}^{\prime}=0.1$ and will be discussed in the next section. Similarly, in Figure $3 b$ the dashed line has a slope of -1.8 and satisfies the condition of continuity for the logarithmic derivative at $\log _{10} r=\log _{10} r_{\mathrm{sw}}=-0.1$. The dash-dot straight line has instead a slope of -1.5 and fits the data points for $\log _{10} r \geq \log _{10} r_{\mathrm{sw}}=0.1$.

For some purposes it is useful to obtain a distribution function $\psi\left(r_{60}^{\prime}\right)$ for just the $60 \mu \mathrm{m}$ flux, where we have introduced the ratio $r_{60}^{\prime}=\left(L_{60} / L_{B}\right)\left(L_{B} / L_{*}\right)^{-\delta^{\prime}}$. The weak correlation between $L_{60} / L_{B}$ and $M_{B}^{0}$ for galaxies in the Virgo and RSA sample gives $\delta^{\prime} \approx 0.05$. For the observed $\psi\left(r_{60}^{\prime}\right)$ we again find that a lognormal curve gives quite a good fit except for the tail for large $r_{60}^{\prime}$. The distribution is a little broader for $r_{60}^{\prime}$ than for $r^{\prime}$, and the best fit for $\psi\left(r_{60}^{\prime}\right)$ gives $\gamma=5.5$ and $r_{0}=0.17$. The theoretical average value of $r_{60}^{\prime}$ over the lognormal curve is then 0.22 . The experimental averages of $r_{60}^{\prime}$, equal to 0.23 for galaxies in $\mathrm{Vo}$ and 0.28 for galaxies in $\mathbf{R b}+\mathbf{R f}$, indicate again the presence of a tail at large values of $r_{60}^{\prime}$.

We can also use the RSA and Virgo data in order to compute the distance-independent FIR color index $c=$ $f_{100} / f_{60}$. The average value for the FIR color index is 2.55 for the Vo sample and 2.75 for the $\mathrm{Rb}$ and $\mathrm{Rf}$ samples together. The scatter diagram of this quantity as a function of the absolute optical magnitude is shown in Figure 4. There is again a weak correlation with the straight line in the figure showing the least-squares fit for $\left\langle\log _{10} c\right\rangle \propto\left(\log _{10} L_{B}\right)^{\epsilon}$ with $\epsilon=0.05 \pm 0.02$. As for the ratio $r$, we define a new variable $c^{\prime}$ by

$$
c^{\prime} \equiv c\left(L_{B} / L_{*}\right)^{-\epsilon} .
$$

The scatter diagram for the two "luminosity-corrected" quantities is shown in Figure 5 (for $\epsilon=0.05$ ). The slope is $-0.17 \pm 0.03$. The relation between the three slopes in Figures 1,4 , and 5 is discussed in $\S 6$.

\section{THE RELATION BETWEEN THE FIR LUMINOSITY FUNCTION AND $\psi(r)$}

In the previous section we saw that the joint optical-FIR distribution function $\Psi\left(L_{B}, r\right)$ is close to a function of a single

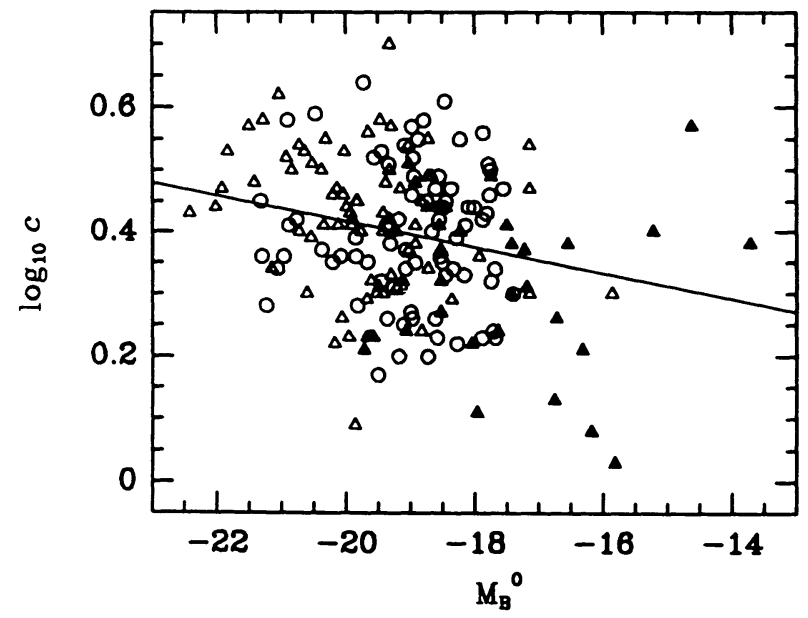

FIG. 4.- Logarithm of the ratio $c=f_{100} / f_{60}$ vs. $M_{B}^{0}$ for all the galaxies in the $\mathrm{Rb}$ sample (open triangles), in the $\mathrm{Rf}$ sample (filled triangles), and in the Vo sample (open circles). The very weak correlation is shown by the straight line, which is the best fit to all the data points in the diagram. 


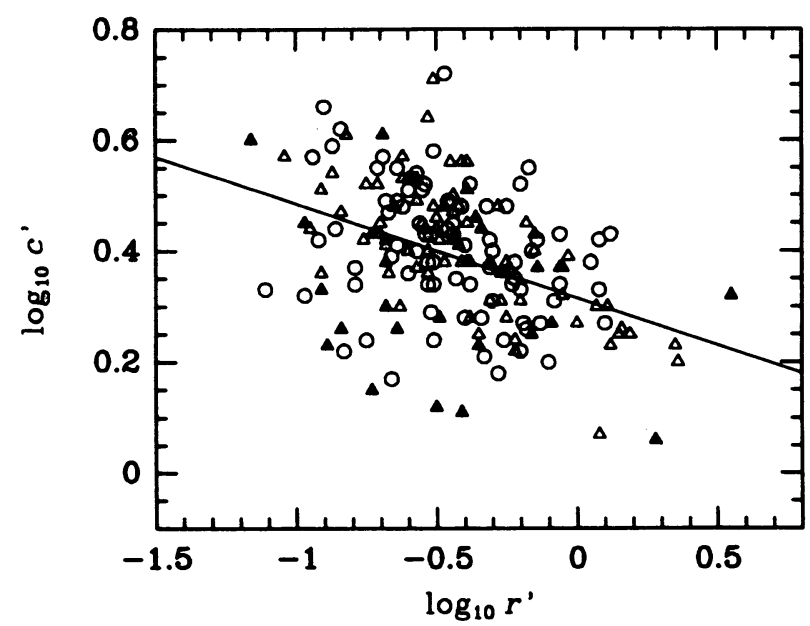

FIG. 5.-Correlation between $\log _{10} c^{\prime}$ and $\log _{10} r^{\prime}$, as defined in the text, for galaxies in the $\mathbf{R b}$ sample (open triangles) for galaxies in the $\mathbf{R f}$ sample (filled triangles), and for galaxies in the Vo sample (open circles). The straight line is the least-squares fit to all the data points.

variable $\psi(r)$ (with minor corrections for faint galaxies). Finding this function requires optically selected data and is now known accurately for small and intermediate values of $r \equiv L_{\mathrm{FIR}} / L_{B}$, but the statistics are poor for studying the small but interesting tail of $\psi$ at very large $r$ (i.e., at very large $L_{\text {FIR }}$ ). On the other hand, an IRAS-selected sample (i.e., a catalog to some flux limit $f_{\text {FIR }}$ ) plus distance measurements gives directly the FIR visibility function,

$$
V\left(L_{\text {FIR }}\right) \propto L_{\text {FIR }}^{1.5} \chi_{\text {FIR }}\left(L_{\text {FIR }}\right),
$$

where $V$ and the FIR luminosity function, $\chi_{\text {FIR }}$, are expressed per logarithmic interval. Because of the factor $L_{\text {FiR }}^{1.5} V$ decreases quite slowly for very large $L_{\mathrm{FIR}}$, and one can explore the tail far out (in contrast to that of $\psi$ ). We shall now derive a relation between $V$ at large $L_{\text {FIR }}$ and $\psi$ at large $r$, which we can use to infer the tail of $\psi$ from the observed tail of $\chi_{\text {FIR }}$ (assuming no evolution of the luminosity function; see Hacking \& Houck 1987).

For most optical galaxy samples (e.g., Efstathiou, Ellis, \& Peterson 1988; Lugger 1989) the optical luminosity function is fitted fairly well by a Schechter function,

$$
\chi_{B}\left(L_{B}\right) \simeq C\left(\frac{L_{B}}{L_{\mathrm{S}}}\right)^{1+\alpha} \exp \left(-\frac{L_{B}}{L_{\mathrm{S}}}\right),
$$

with $C, \alpha$, and $L_{\mathrm{S}}$ constants. For spiral galaxies $\alpha \simeq-1.1$ and $L_{\mathrm{S}} \simeq 5 \times 10^{36} \mathrm{~W}$ (corresponding to $M_{B}^{0}=-20.3$, for $H_{0}=75$ $\mathrm{km} \mathrm{s}^{-1} \mathrm{Mpc}^{-1}$ ), which is about 5 times the typical luminosity $L_{*}$ in our sample described in $\S 3$. We rewrite the integral relation in equation (3) as

$$
\begin{aligned}
V\left(L_{\mathrm{FIR}}\right)=V_{0}\left(\frac{L_{\mathrm{FIR}}}{r_{0} L_{\mathrm{S}}}\right)^{1.5} \int_{-\infty}^{+\infty} \psi\left(\frac{L_{\mathrm{FIR}}}{L_{B}}\right)\left(\frac{L_{B}}{L_{\mathrm{S}}}\right)^{1+\alpha} \\
\quad \times \exp \left(-\frac{L_{B}}{L_{\mathrm{S}}}\right) d\left[\log _{10}\left(\frac{L_{B}}{L_{\mathrm{S}}}\right)\right],
\end{aligned}
$$

where $V_{0}$ is a constant and $\psi$ is assumed to have the form

$$
\psi\left(\frac{L_{\mathrm{FIR}}}{L_{B}}\right)=\psi(r) \propto \begin{cases}\exp \left[-\gamma\left(\log _{10}\left(r / r_{0}\right)\right)^{2}\right] & \text { if } r<r_{\mathrm{sw}}, \\ c_{\mathrm{sw}}\left(r / r_{0}\right)^{-n_{*}} & \text { if } r \geq r_{\mathrm{sw}}\end{cases}
$$

Here $r_{\mathrm{sw}}$ is larger than $r_{0}$, and $c_{\mathrm{sw}}$ is chosen to make $\psi$ (but not necessarily its derivative) continuous at $r=r_{\text {sw }}$. For $L_{\mathrm{FIR}} / L_{\mathrm{S}} \gg$ $r_{\text {sw }}$ the integral can be evaluated approximately, giving the following expression:

$$
V\left(L_{\mathrm{FIR}}\right) \propto \Gamma\left(n_{*}+\alpha+1\right)\left(L_{\mathrm{FIR}} / L_{\mathrm{S}}\right)^{1.5-n_{*}},
$$

where $\Gamma$ is the gamma function,

$$
\Gamma\left(n_{*}+\alpha+1\right) \equiv \int_{0}^{\infty} x^{\alpha+n_{*}} e^{-x} d x .
$$

The function $\Gamma$ does not depend explicitly on $L_{\text {FIR }}$ and depends only weakly on $n_{*}$. For instance, if $\alpha=-1.1$, then $\Gamma=0.89$, 0.91 , and 0.93 , respectively, for $n_{*}=1.5,1.8$, and 1.9. With $n_{*}$ (and hence $\Gamma$ ) constant, $\chi_{\text {FIR }}$ and $\psi$ have the same power-law index for $L_{\text {FIR }} \gg r_{0} L_{\mathrm{S}}$. This is still approximately true if the logarithmic derivative $n(r)$ of $\psi(r)$ is not a constant but varies slowly for $r \gg r_{0}$ : the approximate relation is (with $r_{L} \equiv$ $\left.L_{\text {FIR }} / L_{\mathbf{S}}\right)$

$$
V\left(L_{\mathrm{FIR}}\right) \propto L_{\mathrm{FiR}}^{1.5-n(r L)},
$$

which reduces to equation (13) if $n$ is constant.

We now suggest a "model distribution function" $\psi(r)$ of the analytic form in equation (12), with $\gamma$ and $r_{0}$ based on the observational data in Figure $3 b$ but $n_{*}$ based on data for $\chi_{\text {FIR }}$.

In particular, although $n_{*} \sim(1-1.5)$ has been suggested for $\psi$ at medium-large $r$ (see Rieke \& Lebofsky 1986; RowanRobinson et al. 1987), we prefer $n_{*}$ slightly larger than 1.5. This comes from the analysis not only of data presented in this paper but also of data from a collection of samples presented by Saunders et al. (1990). Moreover, if $n_{*}$ were less than 1.5, then the FIR visibility function $V_{\text {FIR }}\left(L_{\text {FIR }}\right)=\left(L_{\text {FIR }} / r_{0} L_{S}\right)^{1.5} \chi_{\text {FIR }}$ would diverge as $L_{\text {FIR }} \rightarrow \infty$, and in a Euclidean universe a FIR-limited sample would be dominated by very luminous galaxies at very large distances. Cosmological terms would of course cut off the visibility function at high redshifts $z$, but no cutoff should be visible at small $z$. Typical redshifts of starburst galaxies in the Point Source Catalog are $z \lesssim 0.07$ (Lawrence et al. 1986; Soifer et al. 1987; Saunders et al. 1990). Thus the limiting value of $n_{*}$ is probably larger than 1.5 .

We show in Figure $6 a$ the dependence of the observed FIR visibility function on $L_{\mathrm{FIR}} /\left(r_{0} L_{\mathrm{S}}\right)$, where the luminosity function $\chi_{\text {FIR }}$ is taken from Saunders et al. (1990) $\left(L_{\text {FIR }}\right.$ has been corrected to a Hubble constant of $75 \mathrm{~km} \mathrm{~s}^{-1} \mathrm{Mpc}^{-1}$ and multiplied by 2 in order to take into account the emission at $100 \mu \mathrm{m})$. The function plotted in Figure $6 a$ is a broad curve, well represented by a Gaussian in $\log _{10} L_{\text {FIR }}$ for large $L_{\text {FIR }}$ (Saunders et al. 1990). Numerical evaluation of the integral in equation (11) for $n_{*}=1.8\left(\log _{10} r_{\mathrm{sw}}=-0.1\right)$ and $n_{*}=1.5$ $\left(\log _{10} r_{\text {sw }}=-0.1\right)$, as in Figure $3 b$, gives the two predicted visibility functions shown in Figures $6 b$ and $6 c$. The agreement between the observed curve of Saunders et al. (1990) and our predicted visibility function for $n_{*}=1.8$ is tolerably good, especially for $L_{\text {FIR }} /\left(r_{0} L_{\mathrm{S}}\right) \leq 1.5$. The visibility function seems to go down slightly faster for larger values of $L_{\mathrm{FIR}} /\left(r_{0} L_{\mathrm{S}}\right)$. So a power law $r^{-n_{*}}$, with $n_{*} \simeq 1.8$ or slightly larger, gives a good fit not only to the distribution of FIR-optical luminosity of nearby galaxies with large $r$ but also to the FIR luminosity function at larger redshifts.

\section{60 MICRON CLASS AVERAGES FOR A400 AND CANCER}

As seen in Table 2, we have fewer detections at $100 \mu \mathrm{m}$, and we consider only the $60 \mu \mathrm{m}$ data in this section. Besides the 

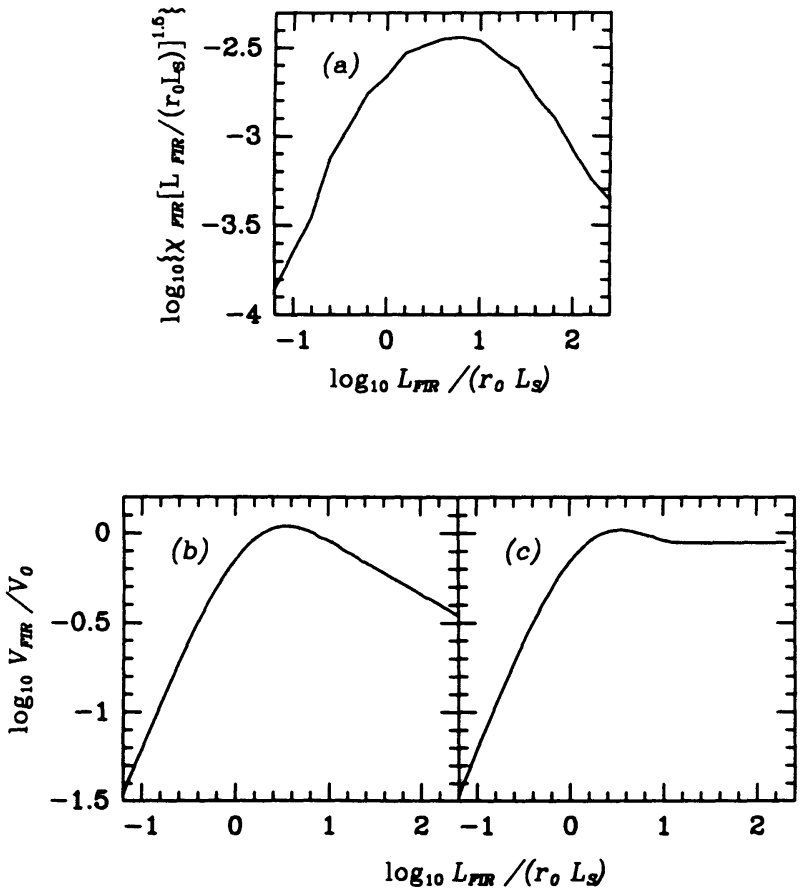

FIG. 6. - (a) Dependence of the observed visibility function $V_{\text {FIR }}$ on $L_{\mathrm{FIR}} /\left(r_{0} L_{S}\right)$; see eq. (9). Data for $\chi_{\mathrm{FIR}}$ are from Saunders et al. (1990) (see text for more details). In $(b)$ and $(c)$ we show the theoretical ratio between the FIR visibility function $V_{\text {FIR }}$ and the constant $V_{0}$ as defined in eq. (11). In (b) we have $n_{*}=1.8\left(\log _{10} r_{\mathrm{sw}}=-0.1\right)$, and in (c) $n_{*}=1.5\left(\log _{10} r_{\mathrm{sw}}=0.1\right)$.

individually detected galaxies for each of the four subgroups (classes) in the A400 and Cancer clusters (Table 2), we have a number $N$ (col. [2] in Table 3) of undetected galaxies. Using the one-dimensional Addscan data on tape obtained from the Infrared Processing and Analysis Center (IPAC), we weighted each undetected galaxy's profile by the inverse of the variance, i.e., the square of the rms noise, and then we averaged the $N$ traces in each of the four classes, keeping the center of each scan at the optical position of each galaxy. Since our class average co-adding averages data obtained from different regions of the sky, the confusion distribution as well as the random noise decreases as $N^{-1 / 2}$, so we expect the rms to be proportional to $N^{-1 / 2}$. Figures $7 a-7 d$ show the class-averaged flux $f_{60}$ plotted against distance $\theta$ from the undetected galaxy position for each of these four classes. The selection of the individual traces had no bias regarding the sign of the flux. The averaged traces in Figure 7, on the other hand, show a clear, narrow positive peak near $\theta=0$ for each of the four classes. The rms noise $\langle\sigma\rangle$ and the averaged $60 \mu \mathrm{m}$ peak flux $\langle f\rangle$ are given in columns (3) and (4) of Table 3.

We can now carry out a statistical comparison with predictions of the $60 \mu \mathrm{m}$ distribution for A400 and Cancer, after

TABLE 3

60 Micron Class Average Data and Estimates

\begin{tabular}{crccccccc}
\hline \hline $\begin{array}{c}\text { Class } \\
(1)\end{array}$ & $\begin{array}{r}N \\
(2)\end{array}$ & $\begin{array}{c}\langle\sigma\rangle \\
(3)\end{array}$ & $\begin{array}{c}\langle f\rangle \\
(4)\end{array}$ & $\begin{array}{c}\log r_{\text {th }}^{\prime} \\
(5)\end{array}$ & $\begin{array}{c}n_{p} \\
(6)\end{array}$ & $\begin{array}{c}n_{e} \\
(7)\end{array}$ & $\begin{array}{c}\log r_{p}^{\prime} \\
(8)\end{array}$ & $\begin{array}{c}\log r_{e}^{\prime} \\
(9)\end{array}$ \\
\hline $\mathrm{Ab} \ldots \ldots \ldots$ & 12 & 0.010 & 0.054 & -1.06 & 0.17 & 0.26 & -1.19 & -1.30 \\
$\mathrm{Af} \ldots \ldots \ldots$. & 30 & 0.009 & 0.045 & -0.48 & 0.83 & 0.76 & -0.80 & -0.81 \\
$\mathrm{Cb} \ldots \ldots \ldots$ & 3 & 0.015 & 0.078 & -1.36 & 0.03 & 0.07 & -1.52 & -1.37 \\
$\mathrm{Cf} \ldots \ldots \ldots$ & 15 & 0.008 & 0.045 & -0.66 & 0.64 & 0.63 & -0.90 & -0.93 \\
\hline
\end{tabular}
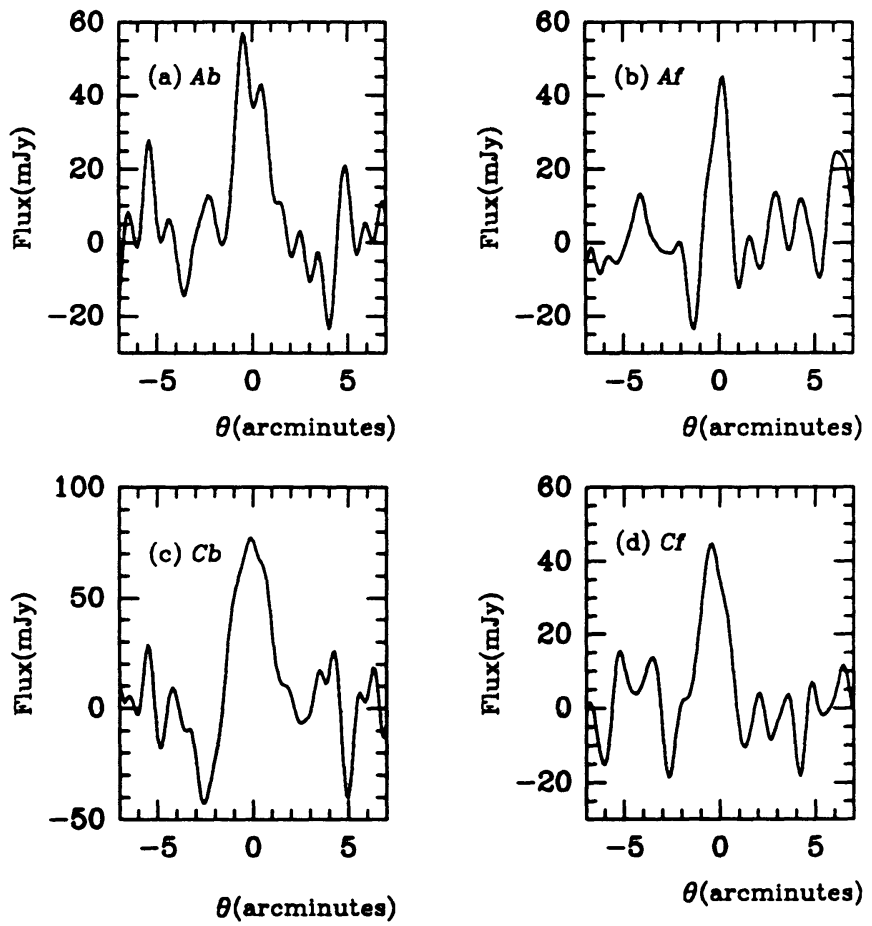

Fig. 7.- Central part of the "class average" spectra at $60 \mu \mathrm{m}$, obtained by averaging the data for the undetected galaxies in each of the groups $\mathrm{Ab}, \mathrm{Af}, \mathrm{Cb}$, Cf.

replacing the individual flux $f_{60}$ of each undetected galaxy by the class average $\langle f\rangle$. We use $r_{60}^{\prime} \equiv\left(L_{60} / L_{B}\right)\left(L_{B} / L_{*}\right)^{-0.05}$, a distance of $100 \mathrm{Mpc}$ (for $H_{0}=75 \mathrm{~km} \mathrm{~s}^{-1} \mathrm{Mpc}^{-1}$ ) for A400, the distances to the various Cancer groups given by Bothum et al. (1983) for galaxies assigned to such groups, and a distance of $64 \mathrm{Mpc}$ to unassigned Cancer galaxies. The solid histograms in Figures $8 a-8 d$ give the contribution of individually detected galaxies to $\psi\left(r_{60}^{\prime}\right)$, the dashed line the contribution of the unde-

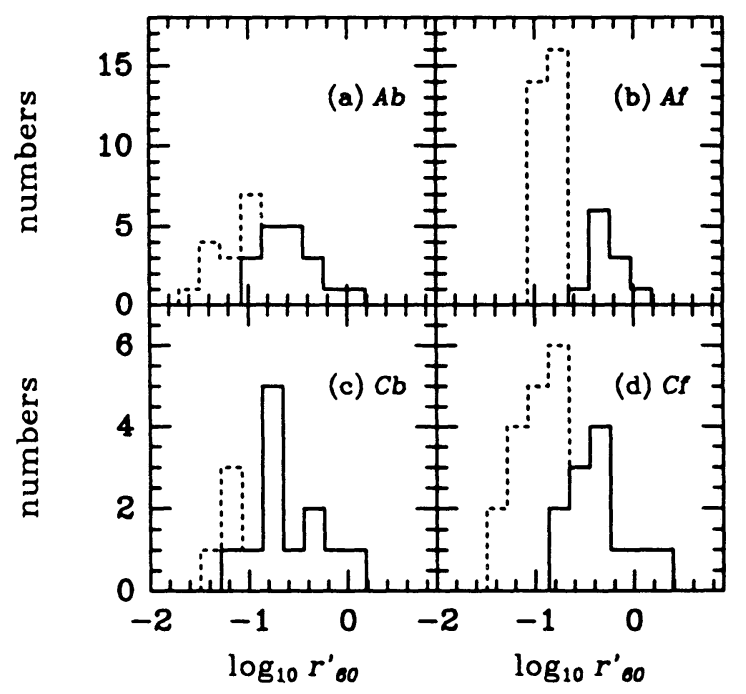

FIG. 8.-Continuous line in $(a),(b),(c)$, and $(d)$ shows the histograms of the logarithm of the ratio $r_{60}^{\prime}$ for all the galaxies detected at $60 \mu \mathrm{m}$ in the Ab, Af, $\mathrm{Cb}, \mathrm{Cf}$ samples, respectively. The dotted line shows $r_{60}^{\prime}$ for the undetected galaxies for which the FIR flux has been set equal to the class average value shown in Table 3. 
tected galaxies with $f$ replaced by $\langle f\rangle$. To compare data with the logarithmic distribution function $\psi\left(r_{60}^{\prime}\right)$, obtained from the RSA and Virgo data, we first determine an average threshold value $r_{\mathrm{th}}^{\prime}$ of $r_{60}^{\prime}$ for detected galaxies in each subgroup. The values for $r_{\mathrm{th}}^{\prime}$, using 3.5 times the weighted rms noise in $f_{60}$ for detected galaxies for the threshold flux, are given in column (5) of Table 3. The predicted fraction $n_{p}$ of all galaxies which have $r_{60}^{\prime}<r_{\text {th }}^{\prime}$, obtained from the integral of the lognormal fit to the distribution function $\psi\left(r_{60}^{\prime}\right)$, is given in column (6), and the actual experimental value $n_{e}$ in column (7). The predicted average value $r_{p}^{\prime}$ of $r_{60}^{\prime}$ for galaxies with $r_{60}^{\prime}<r_{\text {th }}^{\prime}$, derived from the integral of $r_{60}^{\prime} \psi\left(r_{60}^{\prime}\right)$, is given in column (8), and the experimental value $r_{e}^{\prime}$ in column (9). The good agreement with $n_{p}$ and $r_{p}^{\prime}$ shows that the lognormal curve also fits A400 and Cancer at the small end of the range of $r^{\prime}$.

The experimental average value of $r_{60}^{\prime}$ over all galaxies, detected and undetected, is 0.25 and 0.31 for all A400 galaxies $(\mathrm{Ab}+\mathrm{Af})$ and all Cancer galaxies $(\mathrm{Cb}+\mathrm{Cf})$, respectively. This compares fairly well with an average of 0.23 and 0.28 for our Virgo and RSA galaxies, respectively. These values are slightly larger than the average of 0.22 in $\S 3$ for the lognormal curve alone, indicating again the presence of a "tail" at large $r$ '. More generally, our data indicate that the FIR luminosity function does not depend strongly on environment.

\section{SUMMARY AND DISCUSSION}

For optically selected catalogs with data on optical luminosity $L_{B}$ and far-infrared luminosity $L_{\text {FIR }}$, we have discussed the joint distribution function $\Psi\left(L_{B}, r\right)$, which gives, separately for each $L_{B}$, the normalized distribution of the ratio $r=$ $L_{\text {FIR }} / L_{B}$. We used the PSC and co-added data provided by IPAC on spirals and irregular RSA galaxies and the compilation by Helou et al. (1988) on luminous Virgo Cluster galaxies. As noted by previous authors (Bothun et al. 1989), the $r$-distribution does not depend strongly on $L_{B}$ : we do not have enough data to establish any dependence of the shape of the distribution, and the weak dependence of the mean of the distribution can be represented roughly by

$$
\left\langle L_{\mathrm{FIR}} / L_{B}\right\rangle \equiv \int_{-\infty}^{\infty} \Psi\left(L_{B}, r\right) r d r \propto\left(L_{B} / L_{*}\right)^{\delta},
$$

where $L_{*}$ is a typical luminosity for the sample and $\delta$ is small $(\lesssim 0.08)$. The latest Hubble types used in establishing equation (15) are mostly Sm, so we do not know whether there is a deviation from such a power law for the least luminous dwarf irregulars, but there are some qualitative data. As discussed by Helou et al. (1988) and by Hoffman et al. (1989), one must make a distinction between the dwarf irregulars of low surface brightness (LSBDI) and the bright, blue compact dwarfs (BCD galaxies), which show signs of star formation and of low metal abundance. $\left\langle L_{\text {FIR }} / L_{B}\right\rangle$ is particularly small for LSBDI, but for BCD galaxies it is similar to that for luminous spirals. At the lowest $L_{B}$ values, the distribution function is thus likely to be very broad; a value of $\delta$ still near 0.08 or slightly larger may be appropriate there, but data will be required for an extended range of $L_{B}$ to settle this question.

The distribution function $\Psi$, represented by a function of the single variable $\psi\left(r^{\prime}\right)$ with $r^{\prime}=r\left(L_{\mathrm{FIR}} / L_{B}\right)^{-\delta} \simeq r$, is approximately symmetric on a logarithmic scale near its peak (at $r^{\prime}=r_{0}=0.35$ ). As noted before (Rowan-Robinson et al. 1987; Bothun et al. 1989), $\psi\left(r^{\prime}\right)$ near its peak is close to a lognormal curve, equation (7), which is broad but nevertheless falls off steeply on both sides far from the peak. The sensitive co-add data for the nearby RSA and Virgo samples reveals that the actual distribution $\psi$ is indeed steep on the side of low $r^{\prime}$ (every single RSA galaxy has an IRAS detection). This shows that there are very few spiral or irregular galaxies with a very small $r^{\prime}$, i.e., a very low dust-to-gas ratio (we have no "surviving protogalaxies"). By contrast, $\psi$ is known to have a long tail for large $r$, which has been studied best from IR $A S$-selected samples (Rowan-Robinson et al. 1987; Soifer et al. 1987). Most conclusions remain unchanged if $\delta$ is replaced by zero $\left(r^{\prime}=r\right)$. There is no evidence for a double-humped distribution in $\psi$, and a transition from a lognormal curve to a simple power-law tail for $r \gg r_{0}$ (see eq. [12]) gives a reasonable fit. Spirals with peculiar morphology are included in the sample, but we have not investigated how much more common they are in the extreme tail of $\psi$. We showed in equation (14) that the FIR luminosity function, $\chi_{\mathrm{FIR}}$, irrespective of $L_{B}$, is proportional to $\psi$ for large $r$; in particular, $\psi \propto r^{-n_{*}}$ corresponds to $\chi_{\text {FIR }} \propto$ $L_{\text {FIR }}^{-n_{*}}$ for large $L_{\text {FIR }}$.

Without the power-law tail the optical and FIR visibility functions would be similar: The optical luminosity function $\chi_{B}\left(L_{B}\right)$ has a sharp falloff for large $L_{B} / L_{S}$, and Sandage, Binggeli, \& Tammann (1985) showed that the optical visibility function $V_{B}=L_{B}^{1.5} \chi_{B}$ can be represented fairly well as a lognormal curve with dispersion $(1 / 2 \gamma)^{1 / 2}$ of about 0.6 . Convolved with the lognormal function for the ratio $L_{\mathrm{FIR}} / L_{B}$ with dispersion of only 0.28 , this would give a lognormal visibility function $V_{\text {FIR }}$ for $L_{\text {FIR }}$ with a dispersion of about 0.66 , not much larger than that for $L_{B}$. On the other hand, the starburst galaxies in a FIR-selected catalog contribute a particularly slowly varying power-law tail at large $L_{\text {FIR }}$ to the visibility function $V_{\text {FIR }}=$ $L_{\text {FIR }}^{1.5} \chi_{\text {FIR }} \propto L_{\text {FIR }}^{1.5} n_{*}$ (see Fig. 6). This disparity between the two visibility functions also explains a seeming disparity between two different correlations: as seen in equation (6), the distanceindependent ratio $r=L_{\mathrm{FIR}} / L_{B}=F_{\mathrm{FIR}} / F_{B}$ is almost uncorrelated with the absolute optical luminosity $L_{B}$, but (see Rieke \& Lebofsky 1986; Feigelson, Takashi, \& Weedman 1987; Bothu et al. 1989) $r$ correlates quite substantially with the absolute FIR luminosity $L_{\mathrm{FIR}}$. Since $V_{B}$ has no tail, a measurement of apparent optical magnitude $m_{B}$ gives a rough indication of distance; an additional measurement of $r$ distinguishes between normal and starburst galaxies but adds little distance information.

The well-known anticorrelation between $r=L_{\text {FIR }} / L_{B}$ and $c=L_{100} / L_{60}$ (our Fig. 5) has already been explained (Helou 1986; Rowan-Robinson et al. 1987; Rowan-Robinson \& Crawford 1989, hereafter RRC) in terms of large variations in the "burst component" with almost constant "disk component." In particular, RRC note a strong decrease in $L_{60} / L_{B}$ with less change in $L_{100} / L_{B}$ (decrease in $r$ with increase in $c$ ) from S0a to Sc, a portion of the Hubble sequence where $L_{B}$ changes little. On the other hand, our equations (6) and (8) show that (for small $\left.L_{B}\right) r$ and $c$ both decrease (although weakly) with decreasing $L_{B}$, presumably along the Hubble sequence from true spirals to irregulars toward dwarfs. This decrease in $L_{100} / L_{B}$ with little change in $L_{60} / L_{B}$ is probably due to two trends on the faint end of the Hubble sequence (see, e.g., Hoffman et al. 1989): (1) The metal abundance and hence the dust-to-gas ratio decrease toward the dwarfs, so that $L_{\mathrm{FIR}} / L_{\text {opt }}$ for the "disk component" (which emphasizes $L_{100}$ ) decreases (see second to last column in Table 4 of RRC). (2) Increased molecular cloud clumping and star formation toward the dwarfs (some "BCD activity" is already present in Sm galaxies), but with decreased 
metal abundance, can keep the "burst component" (which emphasizes $L_{60}$ ) almost constant.

In $\S 5$ we examined two more distant clusters, the A400 and Cancer clusters, which represent two completely different types of clusters but are at similar redshifts. Using the MAPS, we made an optical catalog of galaxies with absolute magnitude $M_{B}^{0}>-18$, giving a sampie with objects intrinsically as faint as those in the nearest samples (RSA and Virgo) examined in $\$ \$ 3$ and 4 . However, because of the very strong limits on the detectability of faint FIR flux, we are biased in detecting individually only FIR-bright galaxies. In order to overcome this limitation, we have used the one-dimensional data from the Addscan routine for galaxies in the same optical class but individually undetected, after co-adding the scans from IRAS, to evaluate a "class average" FIR flux. Having a large number of galaxies in each class, we were able to lower the noise considerably and to detect average FIR signals at $60 \mu \mathrm{m}$ well below 50 mJy. Table 3 and Figure 7 show reliable $60 \mu \mathrm{m}$ flux averages for each class (Ab, Af, $\mathrm{Cb}, \mathrm{Cf})$. The distribution of FIR emission from disks of normal optical galaxies in these clusters seems quite similar to the results outlined previously for RSA galaxies and galaxies in Virgo. The Cancer Cluster has more gal- axies at the high end of the $L_{\mathrm{FIR}} / L_{B}$ distribution relative to the A400 cluster, and a similar trend is shown by RSA galaxies relative to galaxies in the Virgo Cluster. For small values of $L_{\mathrm{FIR}} / L_{B}$, using the class average results, we have shown that the distribution in $r$ falls off sharply for both $A 400$ and Cancer, compatible with the curve derived in $\S 3$ from nearby data.

We would like to thank the Data Management Team at the Infrared Processing and Analysis Center (IPAC), G. Helou for his helpful suggestions in the data analysis, T. Soifer and J. Houck for discussions, and the referee, M. Rowan-Robinson, for useful suggestions. We also thank Elisa O'Hara for technical help with table preparation, and the Arcetri Observatory in Italy for its hospitality and financial support during this research project. This work was supported in part by the Space Astrophysics Data Analysis Program grant NASA/JPL 957243, and NSF grants AST 87-14475 to Cornell University and AST 87-22990 to the University of Minnesota. The Minnesota Automated Plate Scanner is operated by the University of Minnesota under a grant from the National Science Foundation.
Allen, C. W. 1973, Astrophysical Quantities (London: Athlone) Bicay, M. D., \& Giovanelli, R. 1987, ApJ, 321, 645

Binggeli, B., Sandage, A., \& Tammann, G. A. 1985, AJ, 90, 1985 (VCC)

Bothun, G. D., Geller, M. J., Beers, T. C., \& Huchra, J. P. 1983, ApJ, 268, 47

Bothun, G. D., Lonsdale, C. J., \& Rice, W. 1989, ApJ, 341, 129

Butcher, H. R., \& Oemler, A. J. 1985, ApJS, 57, 665

de Vaucouleurs, G., de Vaucouleurs, A., \& Corwin, H. G. 1976, Second Reference Catalogue of Bright Galaxies (Audtin: University of Texas Press) (RC2) de Vaucouleurs, G., \& Pence, W. D. 1979, ApJS, 40, 425

Dickey, J. M., Keller, D. T., Pennington, R., \& Salpeter, E. E. 1987, AJ, 93, 788

Dressel, L. L., \& Condon, J. J. 1976, ApJS, 31, 187

Dressler, A. 1980, ApJS, 42,565

Efstathiou, G., Ellis, R. S., \& Peterson, B. A. 1988, MNRAS, 232, 431

Feigelson, E. D., Takashu, I., \& Weedman, W. D. 1987, ApJ, 319, L51

Hacking, P., \& Houck, J. R. 1987, ApJS, 63, 311

Helou, G. 1986, ApJ, 311, L33

Helou, G., Khan, I. R., Malek, L., \& Boehmer, L. 1988, ApJS, 68, 151

Hoffman, G. L., Helou, G., Salpeter, E. E., \& Lewis, B. M. 1989, ApJ, 339, 812

IRAS Point Source Catalog, Version 2. 1988, Joint IRAS Science Working Group (Washington, DC: GPO) (PSC)

\section{REFERENCES}

Lawrence, A., Walker, D., Rowan-Robinson, M., Leech, K. J., \& Pentson, M. V. 1986, MNRAS, 219,687

Lugger, P. M. 1989, ApJ, 343, 572

Neugebauer, G., et al. 1984, ApJ, 278, L1

Nilson, P. 1973, Uppsala General Catalogue of Galaxies (Uppsala: Uppsala University Press) (UGC)

Rieke, G. H., \& Lebofsky, M. J. 1986, ApJ, 304, 326

Rowan-Robinson, M., \& Crawford, J. 1989, MNRAS, 238, 523 (RRC)

Rowan-Robinson, M., Helou, G., \& Walker, D. 1987, MNRAS, 227, 589

Sandage, A., Binggeli, B., \& Tammann, G. A. 1985, AJ, 90, 1759

Sandage, A., \& Tammann, G. A. 1981, A Revised Shapley-Ames Catalog of Bright Galaxies (Washington, DC: Carnegie Institution of Washington) (RSA)

Saunders, W., Rowan-Robinson, M., Lawrence, A., Efstathiou, G., Kaiser, N., Ellis, R. S., \& Frenk, C. S. 1990, MNRAS, 242, 318

Soifer, B. T., Sanders, D. B., Madore, B. F., Neugebauer, G., Danielson, G. E Elias, J. H., Lonsdale, C. J., \& Rice, W. L. 1987, ApJ, 320, 238 